\title{
SCALAR CONSERVATION LAWS WITH MULTIPLE ROUGH FLUXES
}

\author{
BENJAMIN GESS AND PANAGIOTIS E. SOUGANIDIS
}

\begin{abstract}
We study pathwise entropy solutions for scalar conservation laws with inhomogeneous fluxes and quasilinear multiplicative rough path dependence. This extends the previous work of Lions, Perthame and Souganidis who considered spatially independent and inhomogeneous fluxes with multiple paths and a single driving singular path respectively. The approach is motivated by the theory of stochastic viscosity solutions which relies on special test functions constructed by inverting locally the flow of the stochastic characteristics. For conservation laws this is best implemented at the level of the kinetic formulation which we follow here.
\end{abstract}

\section{INTRODUCTION}

We are interested in the stochastic scalar conservation law (SSCL for short)

$$
\left\{\begin{array}{l}
d u+\operatorname{div}_{x}(A(x, u) \circ d z)=0 \quad \text { in } \quad \mathbb{R}^{N} \times(0, T), \\
u=u_{0} \quad \text { on } \quad \mathbb{R}^{N} \times\{0\},
\end{array}\right.
$$

where $A$ is a smooth $N \times M$ matrix and $z=\left(z_{1}, \ldots, z_{M}\right)$ is a geometric $\alpha$-Hölder rough path; the precise assumptions are given in Section 2. As a particular example may consider (1.1) with $A$ a diagonal $N \times N$ matrix and $z$ an $N$-dimensional Brownian motion enhanced to a rough path, in which case (1.1) becomes

$$
\left\{\begin{array}{l}
d u+\sum_{j=1}^{N} \partial_{x_{j}} A_{j}(x, u) \circ d z_{j} \quad \text { in } \quad \mathbb{R}^{N} \times(0, T), \\
u=u_{0} \quad \text { on } \quad \mathbb{R}^{N} \times\{0\} .
\end{array}\right.
$$

There is, of course, a close connection with the theory of stochastic viscosity solutions. Indeed when $N=1$ if $v$ solves the stochastic Hamilton-Jacobi equation $d v+A\left(\partial_{x} v, x\right) \circ d z=0$, then, formally, $u=\partial_{x} v$ satisfies the SSCL $d u+\partial_{x}(A(u, x)) \circ d z=0$.

SSCL also arise in several applications. A concrete example is provided by the theory of mean field games developed by Lasry and Lions ( [LL06a, LL06b, LL07]). It turns out that the mean field limit, as $L \rightarrow \infty$, of the empirical law of the solution $\left(X^{1}, \ldots, X^{L}\right) \in \mathbb{R}^{N \times L}$ of the stochastic differential equation

$$
d X_{t}^{i}=\sigma\left(X_{t}^{i}, \frac{1}{L} \sum_{j \neq i} \delta_{X_{t}^{j}}\right) \circ d z_{t} \quad \text { for } i=1, \ldots, L
$$

Date: May 21, 2018.

2000 Mathematics Subject Classification. H6015, 35R60, 35L65.

Key words and phrases. Stochastic scalar conservation laws, rough paths, random dynamical systems, kinetic solutions.

Benjamin Gess has been partially supported by the research project "Random dynamical systems and regularization by noise for stochastic partial differential equations" funded by the German Research Foundation. Panagiotis Souganidis is supported by the NSF grants DMS-0901802 and DMS-1266383. 
where $\sigma$ is a $N \times M$ Lipschitz matrix on $\mathbb{R}^{N} \times \mathcal{P}\left(\mathbb{R}^{N}\right)$ with adjoint $\sigma^{*}, \mathcal{P}\left(\mathbb{R}^{N}\right)$ is the space of probability measures on $\mathbb{R}^{N}$ and $z=\left(z_{1}, \ldots, z_{M}\right)$ is an $N$-dimensional Brownian motion, converges to a measure $\pi_{t}$ which evolves according to

$$
d m+\operatorname{div}_{x}\left[\sigma^{*}(x, m) \circ d z\right]=0 ;
$$

note that (1.3) is not a "standard" conservation law (i.e. of the type (1.2)) unless $\sigma^{*}$ is a diagonal matrix. For this reason we study here SCCL of the more complex type (1.1).

The study of pathwise solutions to SSCL was initiated by Lions, Perthame and Souganidis ( [LPS13]) who introduced the notion of pathwise (stochastic) entropy solutions for "standard" SSCL with $x$ independent fluxes and multiple continuous paths, that is

$$
d u+\sum_{i=1}^{N} \partial_{x_{i}} A_{i}(u) \circ d z_{i}=0,
$$

for $z=\left(z_{1}, \ldots, z_{N}\right)$ continuous. This work was subsequently extended in [LPS14] to $x$-dependent fluxes driven by a single continuous path $z$, that is to SSCL of the form

$$
d u+\sum_{i=1}^{N} \partial_{x_{i}} A_{i}(u, x) \circ d z=0 .
$$

The main contribution of this paper is the extension of the well-posedness theory of the pathwise (stochastic) entropy solutions to general inhomogeneous fluxes with multi-dimensional driving rough paths and non-standard SSCL of the type (1.1).

The theory is based on the general concepts/philosophy around the theory of stochastic viscosity solutions for fully nonlinear first- and second-order PDE including stochastic Hamilton-Jacobi equations which was introduced and developed by Lions and Souganidis in [LS98b, LS98a, LS02, LS00b, LS00a]. The latter is based on a notion of solution which does not depend on the (blowing up) derivatives and integrals of the driving paths. This is accomplished with the introduction of a class of test functions which are short-time smooth solutions of the stochastic Hamilton-Jacobi part of the equation (constructed typically by the method of characteristics) and, when inserted in the equation, at least formally "eliminate" the stochastic part. This is basically a local change of the unknown which however cannot be done unless there is regularity.

The notion of pathwise (stochastic) entropy solutions builds on the above. It is based on the kinetic formulation of conservation laws and test functions which are propagating along the characteristics of the corresponding linear transport equation. The latter are defined globally in time and, in this aspect, the test functions are easier to construct since it is not necessary to invert characteristics. There are, however, new difficulties since the test functions are not easily localizable. For $x$-independent fluxes the characteristics can be solved explicitly and, hence, it is possible to keep track of the cancellations that are taking place. Such explicit solutions are not available when there is spatial dependence. In the presence of one driving force the characteristics can be expressed, after a change of time, in terms of the ones for a conservation law without the rough signals, and this assists in taking care of the cancellations. When dealing with multiple driving paths such expressions are not possible and it becomes necessary to use more sophisticated tools from the rough path theory. In this note we establish the uniqueness and existence of pathwise solutions. For the latter, following [Per02], we prove the existence of what we call generalized pathwise entropy solutions, which are easier to construct using weak limits in the kinetic equations, and then show that they are indeed pathwise solutions. To obtain the weak limits we prove some estimates about the defect measure which are new even in the "deterministic" setting. It is worth remarking that proving strong compactness requires BV-type estimates which are, however, not at all obvious due to the inhomogeneity of the equations 
and the singularities of the paths. Such estimates were obtained in [LPS13] but there the fluxes were $x$-independent.

As already outlined above, the concept of pathwise rough entropy/kinetic solutions builds upon the characteristic system corresponding to the kinetic formulation. Due to the roughness of the driving signal $z$ the this system becomes a stochastic differential equation (SDE). In order to justify this notion of a solution and to construct solutions we will consider approximating problems based on smoothed signals $z^{n} \in C^{\infty}\left([0, T] ; \mathbb{R}^{M}\right)$. An essential ingredient of the construction will thus be the continuous dependence of the characteristics on the driving signal $z$. However, it is well-known that in general SDE do not depend continuously on the paths of the driving signal. This is where rough paths theory enters the picture. The relevance of rough paths in this work is that they provide stronger topologies on the space of the driving signals making the solution to SDE, in our case the system of characteristics, continuous with respect to the driving signal.

We remark that the term "stochastic" may not be the best choice to characterize the solutions we study which rely on rough paths and use very little if at all stochasticity. A better name, which we use here, is pathwise rough entropy/kinetic solutions. To avoid any confusion we emphasize that the solutions we study here coincide with the ones in [LPS13,LPS14] when considering the setting of these references.

Recently Debussche and Vovelle [DV10, Feng and Nualart FN08 and Chen, Ding and Karlsen CDK12 (see also Debussche, Hofmanova and Vovelle [DHV13] and Hofmanova [Hof13]) have put forward a theory of weak entropy solutions of scalar conservation laws with Itô-type semilinear and not quasilinear stochastic dependence. Such problems do not appear amenable to a pathwise theory. Our results do not cover the equations studied in [DV10, FN08] and vice versa. We refer to Section 6 of [LPS13] for a discussion of these issues. A rough paths approach to SSCL with linear transport noise has been developed in [FG14].

We conclude the introduction emphasing that this paper builds upon the ideas of [LPS13, LPS14 and, of course, the general methodology of the work on stochastic viscosity solutions [LS98b, LS98a, LS02, LS00b, LS00a, We expect that several extensions may be possible without a real change in the general strategy. For example, it should take only few and straightforward technical modifications to extend the results to equations like (1.1) plus a semilinear term. We leave such extensions to the interested reader.

Organization of the paper. The paper is organized as follows: In Section 2 we state the assumptions, we review briefly some facts about entropy solutions and their kinetic formulation and we introduce the notion of pathwise rough entropy/kinetic solutions. Their uniqueness is proved in Section 3, a key step in the proof is a technical lemma about solutions of differential equations with rough paths which is presented in the Appendix. The construction of pathwise rough entropy/kinetic solutions, which requires some some new estimates, is presented in Section 4. In the two Appendices we recall some facts from the theory of rough paths used in the main body of the paper and prove an estimate that is used throughout the paper.

Notation and terminology. We work in the $N$-dimensional Euclidean space $\mathbb{R}^{N}$. If $x \in \mathbb{R}^{N}$ and $\xi \in \mathbb{R},\left\|\begin{array}{l}x \\ \xi\end{array}\right\|$ denotes the norm of $(x, \xi)$ in $\mathbb{R}^{N+1}$. If $b=\left(b^{1}, \ldots, b^{N}\right): \mathbb{R}^{N} \rightarrow \mathbb{R}^{N}$ is a smooth vector field, divb $:=\sum_{i=1}^{N} \partial_{x_{i}} b^{i}$. We say that $A \subset[0, T]$ is null if it has zero Lebesgue measure. The space of bounded measures in $\mathbb{R}^{N} \times \mathbb{R}$ is denoted by $\mathcal{M}\left(\mathbb{R}^{N} \times \mathbb{R}\right)$. For a set $C \subset[0, T]$ we define $\Delta(C):=\{(s, t)$ : $s, t \in C, s \leq t\}$. For $\delta>0, \operatorname{Lip}^{\delta}\left(\mathbb{R}^{N} \times \mathbb{R} ; \mathbb{R}^{N}\right)$ is the set of functions with $k=0, \ldots\lfloor\delta\rfloor$ bounded derivatives and $\delta-\lfloor\delta\rfloor$ Hölder continuous $\lfloor\delta\rfloor$-th derivative. If $\Delta=\left\{a=t_{0} \leq t_{i} \leq \ldots \leq t_{M}=b\right\}$ is a partition of $[a, b] \subset[0, T]$, then $\|\Delta\|:=\left\{\left|t_{i+1}-t_{1}\right|: i=0, \ldots, M-1\right\}$. 


\section{The Kinetic Formulation And PATHWise ROUGH ENTROPy/KINETIC SOlutions}

Assumptions. We present the assumptions we need to make to study (1.1).

The first is that, for some $\alpha \in(0,1)$,

$$
z \text { is an } \alpha \text {-Hölder geometric rough path, }
$$

that is $z \in C^{0, \alpha}\left([0, T] ; G^{\left\lfloor\frac{1}{\alpha}\right\rfloor}\left(\mathbb{R}^{N}\right)\right)$. For some background on rough paths and the meaning of $G^{\left\lfloor\frac{1}{\alpha}\right\rfloor}\left(\mathbb{R}^{N}\right)$ we refer to the Appendix $\mathrm{A}$.

As as far as the flux is concerned we assume that, for some $M \in \mathbb{N}$,

$$
A \in C^{1}\left(\mathbb{R}^{N} \times \mathbb{R} ; \mathbb{R}^{N \times M}\right) .
$$

We consider the matrix $a \in \mathbb{R}^{N \times M}$ and vector $b \in \mathbb{R}^{M}$ given by

$$
a(x, \xi)=: A_{\xi}(x, \xi) \in \mathbb{R}^{N \times M} \text { and } b(x, \xi)=: \operatorname{div} A(x, \xi) ;
$$

note that the $(i j)$-element $a^{i j}$ of the matrix $a(x, \xi)$ is $A_{\xi}^{i j}(x, \xi)$ and $(\operatorname{div} A)^{j}:=\sum_{i=1}^{N} \partial_{x_{i}} A^{i j}$, where, for $i=1, \ldots, N$ and $j=1, \ldots, M, A^{i j}$ is the $(i j)$ element of $A$.

We assume that, for some $\gamma>\frac{1}{\alpha} \geq 1$,

$$
a, b \in \operatorname{Lip}^{\gamma+2}\left(\mathbb{R}^{N} \times \mathbb{R}\right),
$$

and, for all $x \in \mathbb{R}^{N}$,

$$
b(x, 0)=0 .
$$

Kinetic formulation. The well established theories of entropy solutions and kinetic solutions (see, for example, Dalibard Dal06] and Perthame Per02]) extend easily to problems with smooth driving paths, that is for $z \in C^{1}\left([0, T] ; \mathbb{R}^{M}\right)$. With this assertion at hand, below we recall the basic facts. Given the nonlinear function

$$
\chi(x, \xi, t):=\chi(u(x, t), \xi):=\left\{\begin{array}{l}
+1 \text { if } 0 \leq \xi \leq u(x, t), \\
-1 \text { if } u(x, t) \leq \xi \leq 0, \\
0 \text { otherwise }
\end{array}\right.
$$

we may rewrite (1.1) in its kinetic form. Recalling that here we are assuming smooth driving signals, to simplify the notation we write

$$
a_{i}(x, \xi, t):=\sum_{j=1}^{M}\left(\partial_{u} A^{i, j}\right)(x, \xi) \dot{z}^{j}(t)
$$

and

$$
b(x, \xi, t):=\sum_{i=1}^{N} \sum_{j=1}^{M}\left(\partial_{x_{i}} A^{i, j}\right)(x, \xi) \dot{z}^{j}(t)=\operatorname{div} A(x, \xi) \cdot \dot{z}(t) .
$$

In view of (2.4) we have

$$
b(x, 0, t)=b(x, 0) \cdot \dot{z}(t)=0 .
$$

Fix $T>0$. The kinetic form of (1.1) is

$$
\left\{\begin{array}{l}
\partial_{t} \chi+a(x, \xi, t) \cdot D_{x} \chi-b(x, \xi, t) \partial_{\xi} \chi=\partial_{\xi} m \text { in } \mathbb{R}^{N} \times \mathbb{R} \times(0, T), \\
\chi=\chi\left(u_{0}(\cdot), \cdot\right) \text { on } \mathbb{R}^{N} \times \mathbb{R} \times\{0\}
\end{array}\right.
$$


where $m$ is a bounded nonnegative measure on $\mathbb{R}^{N} \times \mathbb{R} \times[0, T]$;

the precise bounds on the mass of the measure are stated later.

Due to the Hamiltonian structure of (2.6), we may rewrite it as

$$
\left\{\begin{array}{l}
\partial_{t} \chi+\operatorname{div}_{x}(a(x, \xi, t) \chi)-\partial_{\xi}(b(x, \xi, t) \chi)=\partial_{\xi} m \text { in } \mathbb{R}^{N} \times \mathbb{R} \times(0, T), \\
\chi=\chi\left(u_{0}(\cdot), \cdot\right) \text { on } \mathbb{R}^{N} \times \mathbb{R} \times\{0\} .
\end{array}\right.
$$

Derivation of a stable notion of kinetic solutions. As stated above the notion of kinetic solutions is not well defined for rough driving signals, since the coefficients $a, b$ blow up with $\dot{z}$. On the other hand, following [LPS13, [LPS14, we observe that the linearity of (2.6) in $\chi$ suggests that we may use the characteristics of (2.6) to derive a stable notion of solution, which we will call pathwise rough entropy/kinetic solutions.

For now we continue assuming that $z \in C^{1}\left([0, T] ; \mathbb{R}^{M}\right)$ and, for every $t_{0} \in[0, T]$, we consider the corresponding forward/backward transport equation

$$
\left\{\begin{array}{l}
\partial_{t} \varrho_{t_{0}}+a(x, \xi, t) \cdot D_{x} \varrho_{t_{0}}-b(x, \xi, t) \partial_{\xi} \varrho_{t_{0}}=0 \text { in } \mathbb{R}^{N} \times \mathbb{R} \times \mathbb{R}, \\
\varrho_{t_{0}}=\varrho^{0} \text { on } \mathbb{R}^{N} \times \mathbb{R} \times\left\{t_{0}\right\},
\end{array}\right.
$$

which is rewritten as

$$
\left\{\begin{array}{l}
\partial_{t} \varrho_{t_{0}}+\sum_{i=1}^{N} \sum_{j=1}^{M}\left(\partial_{u} A^{i, j}\right)(x, \xi) \partial_{x_{i}} \varrho_{t_{0}} \dot{z}^{j}(t)-\sum_{i=1}^{N} \sum_{j=1}^{M}\left(\partial_{x_{i}} A^{i, j}\right)(x, \xi) \partial_{\xi} \varrho_{t_{0}} \dot{z}^{j}(t)=0 \\
\varrho_{t_{0}}\left(t_{0}\right)=\varrho^{0} .
\end{array}\right.
$$

We note that in view of the linearity of (2.8) and (2.9), it makes sense to consider solutions starting at $t_{0}$ and existing forward and backward in time.

For each $(y, \eta) \in \mathbb{R}^{N+1}, t_{0} \geq 0$ and $\varrho^{0} \in C_{c}^{\infty}\left(\mathbb{R}^{N} \times \mathbb{R}\right)$ we consider the solution $\varrho_{t_{0}}=\varrho_{t_{0}}(x, y, \xi, \eta, t)$ to (2.8) with $\varrho_{t_{0}}\left(\cdot, y, \cdot, \eta, t_{0}\right)=\varrho^{0}(\cdot-y, \cdot-\eta)$. Defining the convolution along characteristics by

$$
\varrho_{t_{0}} * \chi(y, \eta, t):=\int \varrho_{t_{0}}(x, y, \xi, \eta, t) \chi(x, \xi, t) d x d \xi,
$$

we find (see Lemma 2.3 below)

$$
\partial_{t} \varrho_{t_{0}} * \chi(y, \eta, t)=-\int \partial_{\xi} \varrho_{t_{0}}(x, y, \xi, \eta, t) m(x, \xi, t) d x d \xi \text { in } \mathbb{R}^{N} \times \mathbb{R} \times(0, T) .
$$

The characteristics. It is a classical fact that the solution of (2.8) can be expressed in terms of the associated (backward) characteristics starting at $t_{0} \geq 0$. Note that, in contrast to (2.8), the characteristic equations are well-defined also for rough driving signals $z$.

We assume next that $z$ is an $\alpha$-Hölder geometric rough path and we consider, for $i=1, \ldots, N$, the rough differential equations

$$
\left\{\begin{array}{l}
d Y_{\left(t_{0}, y, \eta\right)}^{i}(t)=\sum_{j=1}^{M} a^{i, j}\left(Y_{\left(t_{0}, y, \eta\right)}(t), \zeta_{\left(t_{0}, y, \eta\right)}(t)\right) d z^{j}(t), \quad Y_{\left(t_{0}, y, \eta\right)}^{i}\left(t_{0}\right)=y^{i}, \\
d \zeta_{\left(t_{0}, y, \eta\right)}(t)=-\sum_{i=1}^{N} \sum_{j=1}^{M}\left(\partial_{x_{i}} A^{i, j}\right)\left(Y_{\left(t_{0}, y, \eta\right)}(t), \zeta_{\left(t_{0}, y, \eta\right)}(t)\right) d z^{j}(t), \quad \zeta_{\left(t_{0}, y, \eta\right)}\left(t_{0}\right)=\eta .
\end{array}\right.
$$

We note that, in light of (2.3) and [FV10], there exits a unique solution to (2.10) and, for $Y_{\left(t_{0}, y, \eta\right)}(t):=$ $\left(Y_{\left(t_{0}, y, \eta\right)}^{1}(t), \ldots, Y_{\left(t_{0}, y, \eta\right)}^{N}(t)\right)$, the map

$$
\left(\begin{array}{c}
Y_{t_{0}} \\
\zeta_{t_{0}}
\end{array}\right)(t):\left(\begin{array}{c}
y \\
\eta
\end{array}\right) \mapsto\left(\begin{array}{c}
Y_{\left(t_{0}, y, \eta\right)}(t) \\
\zeta_{\left(t_{0}, y, \eta\right)}(t)
\end{array}\right)
$$

is a flow of homeomorphisms on $\mathbb{R}^{N+1}$. 
For $t \geq t_{0}$ set

$$
\left(\begin{array}{c}
Y_{(t, y, \eta)}\left(t_{0}\right) \\
\zeta_{(t, y, \eta)}\left(t_{0}\right)
\end{array}\right):=\left[\left(\begin{array}{c}
Y_{t_{0}} \\
\zeta_{t_{0}}
\end{array}\right)(t)\right]^{-1}\left(\begin{array}{c}
y \\
\eta
\end{array}\right)
$$

and observe that, in view of (2.4),

$$
\zeta_{\left(t_{0}, y, 0\right)} \equiv 0
$$

and, hence, for all $t$,

$$
\operatorname{sgn}\left(\zeta_{\left(t_{0}, y, \eta\right)}(t)\right)=\operatorname{sgn}(\eta)
$$

For each time $t_{1} \geq 0$ and for $i=1, \ldots, N$ we consider the backward characteristics

$$
\begin{aligned}
& d X_{\left(t_{1}, x, \xi\right)}^{i}(t)=\sum_{j=1}^{M} a^{i, j}\left(X_{\left(t_{1}, x, \xi\right)}(t), \Xi_{\left(t_{1}, x, \xi\right)}(t)\right) d z^{t_{1}, j}(t), \\
& d \Xi_{\left(t_{1}, x, \xi\right)}(t)=-\sum_{i=1}^{N} \sum_{j=1}^{M}\left(\partial_{x_{i}} A^{i, j}\right)\left(X_{\left(t_{1}, x, \xi\right)}(t), \Xi_{\left(t_{1}, x, \xi\right)}(t)\right) d z^{t_{1}, j}(t), \\
& X_{\left(t_{1}, x, \xi\right)}^{i}(0)=x^{i} \text { and } \Xi_{\left(t_{1}, x, \xi\right)}(0)=\xi .
\end{aligned}
$$

where, for $t \in\left[0, t_{1}\right], z^{t_{1}}$ is the time-reversed rough path, that is

$$
z^{t_{1}}(t):=z\left(t_{1}-t\right) \text {. }
$$

Then, for all $t \in\left[t_{0}, t_{1}\right]$, we have

$$
X_{\left(t_{1}, Y_{\left(t_{0}, y, \eta\right)}\left(t_{1}\right), \zeta_{\left(t_{0}, y, \eta\right)}\left(t_{1}\right)\right)}\left(t_{1}-t\right)=Y_{\left(t_{0}, y, \eta\right)}(t)
$$

and

$$
\Xi_{\left(t_{1}, Y_{\left(t_{0}, y, \eta\right)}\left(t_{1}\right), \zeta_{\left(t_{0}, y, \eta\right)}\left(t_{1}\right)\right)}\left(t_{1}-t\right)=\Xi_{\left(t_{0}, y, \eta\right)}(t) .
$$

In particular, the inverse of the homeomorphism $\left(\begin{array}{c}Y_{t_{0}} \\ \zeta_{t_{0}}\end{array}\right)\left(t_{1}\right)$ is given by

$$
\left(\begin{array}{c}
Y_{\left(t_{1}, x, \xi\right)}\left(t_{0}\right) \\
\zeta_{\left(t_{1}, x, \xi\right)}\left(t_{0}\right)
\end{array}\right)=\left[\left(\begin{array}{c}
Y_{t_{0}} \\
\zeta_{t_{0}}
\end{array}\right)\left(t_{1}\right)\right]^{-1}\left(\begin{array}{c}
x \\
\xi
\end{array}\right)=\left(\begin{array}{c}
X_{\left(t_{1}, x, \xi\right)}\left(t_{1}-t_{0}\right) \\
\Xi_{\left(t_{1}, x, \xi\right)}\left(t_{1}-t_{0}\right)
\end{array}\right)
$$

Hence, the solution $\varrho_{t_{0}}$ to (2.8) with $\varrho_{t_{0}}\left(\cdot, y, \cdot, \eta, t_{0}\right)=\varrho^{0}(\cdot-y, \cdot-\eta)$ is given, for all $t \in[0, T]$, by

$$
\varrho_{t_{0}}(x, y, \xi, \eta, t)=\varrho^{0}\left(\begin{array}{c}
X_{(t, x, \xi)}\left(t-t_{0}\right)-y \\
\Xi_{(t, x, \xi)}\left(t-t_{0}\right)-\eta
\end{array}\right)=\varrho^{0}\left(\begin{array}{c}
Y_{(t, x, \xi)}\left(t_{0}\right)-y \\
\zeta_{(t, x, \xi)}\left(t_{0}\right)-\eta
\end{array}\right) .
$$

Pathwise rough entropy/kinetic solutions. We introduce the definition of the pathwise rough entropy/kinetic solution.

Definition 2.1. Let $u_{0} \in\left(L^{1} \cap L^{2}\right)\left(\mathbb{R}^{N}\right)$. A function $u \in L^{\infty}\left([0, T] ; L^{1}\left(\mathbb{R}^{N}\right)\right)$ is a pathwise rough entropy/kinetic solution to (1.1), if there exists a nonnegative bounded measure $m$ on $\mathbb{R}^{N} \times \mathbb{R} \times[0, T]$ such that, for all $t_{0} \geq 0$, all test functions $\varrho_{t_{0}}$ given by $(2.12)$ with $\varrho^{0} \in C_{c}^{\infty}\left(\mathbb{R}^{N+1}\right)$ and $\varphi \in C_{c}^{\infty}([0, T))$,

$$
\int_{0}^{T} \partial_{t} \varphi(r)\left(\varrho_{t_{0}} * \chi\right)(y, \eta, r) d r+\varphi(0)\left(\varrho_{t_{0}} * \chi\right)(y, \eta, 0)=\int_{0}^{T} \int \varphi(r) \partial_{\xi} \varrho_{t_{0}}(x, y, \xi, \eta, r) m(x, \xi, t) d x d \xi d r .
$$

Throughout the paper we will use a different almost pointwise in form of the definition. Since this will be used several times in the paper, we state it as a separate Proposition. 
Proposition 2.2. Let $u_{0}, m$ be as in Definition 2.1. Then $u \in L^{\infty}\left([0, T] ; L^{1}\left(\mathbb{R}^{N}\right)\right)$ is a pathwise rough entropy/kinetic solution to (1.1) if and only if there exists a null set $\mathcal{N} \subseteq[0, T]$ such that $0 \notin \mathcal{N}$ and, for all $t_{0} \geq 0,(s, t) \in \Delta([0, T] \backslash \mathcal{N})$ and $(y, \eta) \in \mathbb{R}^{N+1}$ and all test functions $\varrho_{0}$ given by (2.12) with $\varrho^{0} \in C_{c}^{\infty}\left(\mathbb{R}^{N+1}\right)$,

$$
\varrho_{t_{0}} * \chi(y, \eta, t)-\varrho_{t_{0}} * \chi(y, \eta, s)=-\int_{s}^{t} \int \partial_{\xi} \varrho_{t_{0}}(x, y, \xi, \eta, r) m(x, \xi, r) d x d \xi d r
$$

Note that applying Lebesgue's differentiation theorem both for right-handed and centered averages yields that the entropy defect measure $m$ does not have point masses on $[0, T] \backslash \mathcal{N}$. In particular, the integrals appearing in (2.13) are well-defined.

Proof. An application of Lebesque's differentiation theorem implies the claim for a null $\mathcal{N} \subseteq[0, T]$ possibly depending on $t_{0}$ and $\varrho^{0}$. We then choose countable dense subset $A \subseteq[0, T]$ and a countable $B \subseteq C_{c}^{\infty}\left(\mathbb{R}^{N+1}\right)$ such that, for each $\varrho^{0} \in C_{c}^{\infty}\left(\mathbb{R}^{N+1}\right)$, there exists a sequence $\varrho^{0, n} \in B$ with uniformly compact support, such that $\varrho^{0, n} \rightarrow \varrho^{0}$ in $C^{1}\left(\mathbb{R}^{N+1}\right)$.

Since $A, B$ are countable, there exists a null $\mathcal{N} \subseteq[0, T]$ such that (2.13) holds for all $t_{0} \in A, \varrho^{0} \in B$. For arbitrary $t_{0} \in[0, T], \varrho^{0} \in C_{c}^{\infty}\left(\mathbb{R}^{N+1}\right)$ we may then choose approximating sequences $t_{0}^{n} \in A$, $\varrho^{0, n} \in B$ with $t_{0}^{n} \rightarrow t, \varrho^{0, n} \rightarrow \rho$ in $C^{1}$ and $\varrho^{0, n}$ having uniformly compact support. Then (2.13) is satisfied for all $t_{0}^{n}, \varrho^{0, n}$, that is

$$
\varrho_{t_{0}^{n}}^{n} * \chi(y, \eta, t)-\varrho_{t_{0}^{n}}^{n} * \chi(y, \eta, s)=-\int_{s}^{t} \int \partial_{\xi} \varrho_{t_{0}^{n}}^{n}(x, y, \xi, \eta, r) m(x, \xi, r) d x d \xi d r
$$

In view of (2.12) and the continuity in time of the characteristics $(X, \Xi)$ (see Appendix $\mathrm{A}$ ) we may take the limit $n \rightarrow \infty$ in the left hand side of (2.14). As for the right hand side goes we note that

$$
\begin{aligned}
\partial_{\xi} \varrho_{t_{0}^{n}}^{n}(x, y, \xi, \eta, t) & =D_{x} \varrho^{0, n}\left(\begin{array}{c}
X_{(t, x, \xi)}\left(t-t_{0}^{n}\right)-y \\
\Xi_{(t, x, \xi)}\left(t-t_{0}^{n}\right)-\eta
\end{array}\right) \partial_{\xi} X_{(t, x, \xi)}\left(t-t_{0}^{n}\right) \\
& +\partial_{\xi} \varrho^{0, n}\left(\begin{array}{c}
X_{(t, x, \xi)}\left(t-t_{0}^{n}\right)-y \\
\Xi_{(t, x, \xi)}\left(t-t_{0}^{n}\right)-\eta
\end{array}\right) \partial_{\xi} \Xi_{(t, x, \xi)}\left(t-t_{0}^{n}\right) .
\end{aligned}
$$

The continuity of the characteristics yields

$$
\partial_{\xi} \varrho_{t_{0}^{n}}^{n}(x, y, \xi, \eta, t) \rightarrow \partial_{\xi} \varrho_{t_{0}}(x, y, \xi, \eta, t) \text { in } C^{1}\left(\mathbb{R}^{N+1}\right)
$$

Since the $\varrho^{0, n}$ 's have uniformly compact support, we may thus take the limit $n \rightarrow \infty$ in the right hand side of (2.14) to conclude.

Next we show that, for smooth paths, the notions of kinetic and pathwise rough entropy/kinetic solutions are equivalent.

Lemma 2.3. Assume that $z \in C^{1}\left([0, T] ; \mathbb{R}^{N}\right)$ and $u \in L^{\infty}\left([0, T] ; L^{1}\left(\mathbb{R}^{N}\right)\right)$. Then $u$ is a pathwise rough entropy/kinetic solution to (1.1) if and only if $u$ is a kinetic solution.

Proof. Let $u$ be a kinetic solution to (1.1). Then, for all $\varphi \in C_{c}^{1}\left(\mathbb{R}^{N} \times \mathbb{R} \times[0, T)\right)$,

$\int_{0}^{T} \int \chi\left(\partial_{t} \varphi+a(x, \xi, r) \cdot D_{x} \varphi-b(x, \xi, r) \partial_{\xi} \varphi\right) d \xi d x d r+\int \chi(x, \xi, 0) \varphi(x, \xi, 0) d \xi d x=\int_{0}^{T} \int m \partial_{\xi} \varphi d \xi d x d r$. 
It follows that there exists a null set $\mathcal{N} \subseteq[0, T]$ such that $0 \notin \mathcal{N}$ and, for all $(s, t) \in \Delta([0, T] \backslash \mathcal{N})$ and all $\varphi \in C_{c}^{1}\left(\mathbb{R}^{N} \times \mathbb{R} \times[0, T]\right)$,

$$
\begin{aligned}
& \int_{s}^{t} \int \chi(x, \xi, r)\left(\partial_{t} \varphi(x, \xi, r)+a(x, \xi, r) \cdot D_{x} \varphi(x, \xi, r)-b(x, \xi, r) \partial_{\xi} \varphi(x, \xi, r)\right) d \xi d x d r+ \\
& \int \chi(x, \xi, t) \varphi(x, \xi, t) d \xi d x-\int \chi(x, \xi, s) \varphi(x, \xi, s) d \xi d x=\int_{s}^{t} \int m \partial_{\xi} \varphi d \xi d x d r .
\end{aligned}
$$

Choosing $\varphi=\varrho_{t_{0}}$ yields

$$
\int \chi(x, \xi, t) \varrho_{t_{0}}(x, y, \xi, \eta, t) d \xi d x-\int \chi(x, \xi, s) \varrho_{t_{0}}(x, y, \xi, \eta, s) d \xi d x=\int_{s}^{t} \int m \partial_{\xi} \varrho_{t_{0}} d \xi d x d r
$$

which by Remark 2.2 implies that $\chi$ is a pathwise rough entropy/kinetic solution to (1.1).

In Theorem 3.1 below we prove the uniqueness of pathwise rough entropy/kinetic solutions. Combined with the existence of kinetic solutions (see [Dal06, Per02]) this implies that every pathwise rough entropy/kinetic solution is a "classical" kinetic solution.

\section{The uniqueness of PATHWise Rough Entropy/Kinetic SOlutions}

We discuss here the following theorem that yields the uniqueness of pathwise rough entropy/kinetic solutions.

Theorem 3.1. Let $u^{(1)}, u^{(2)} \in L^{\infty}\left([0, T] ; L^{1}\left(\mathbb{R}^{N}\right)\right)$ be two pathwise rough entropy/kinetic solutions to (1.1) with initial values $u_{0}^{1}, u_{0}^{2} \in\left(L^{1} \cap L^{2}\right)\left(\mathbb{R}^{N}\right)$. Then, for a.e. $t \in[0, T]$,

$$
\left\|u^{(1)}(t)-u^{(2)}(t)\right\|_{L^{1}\left(\mathbb{R}^{d}\right)} \leq\left\|u_{0}^{1}-u_{0}^{2}\right\|_{L^{1}\left(\mathbb{R}^{d}\right)} .
$$

Before we present the details, we describe briefly the main idea of the proof and motivate some of the technicalities. The key observation, that goes back to [Per02], is that

$$
\begin{aligned}
\left\|u^{(1)}(t)-u^{(2)}(t)\right\|_{L^{1}\left(\mathbb{R}^{d}\right)} & =\int\left(\chi^{(1)}(x, \xi, t)-\chi^{(2)}(x, \xi, t)\right)^{2} d x d \xi \\
& =\int\left|\chi^{(1)}(x, \xi, t)\right|+\left|\chi^{(2)}(x, \xi, t)\right|-2 \chi^{(1)}(x, \xi, t) \chi^{(2)}(x, \xi, t) d x d \xi
\end{aligned}
$$

where, for $i=1,2, \chi^{(i)}$ is related to $u^{(i)}$ by (2.5). To obtain the contraction estimate (3.1), it then suffices, in principle, to show that the derivative with respect to $t$ of the right hand side of the above equality is nonpositive; actually this is how uniqueness is shown for conservation laws with smooth time dependence. The difficulty here is that it is not possible to perform this differentiation due to the lack of regularity. Following instead [LPS13, LPS14 we replace $\chi^{(1)}, \chi^{(2)}$ by $\varrho_{t_{0}} * \chi^{(1)}, \varrho_{t_{0}} * \chi^{(2)}$ for suitable choices of $\varrho_{t_{0}}$. Then it is possible to differentiate with respect to $t$ at the expense of creating several additional terms that need to be estimated. 
Proof of Theorem 3.1. For a fixed $t_{0} \in[0, T]$ we have

$$
\begin{aligned}
& -2 \frac{d}{d t} \int\left(\chi^{(1)} * \varrho_{t_{0}}\right)\left(\chi^{(2)} * \varrho_{t_{0}}\right) d y d \eta \\
& =-2 \int\left(\chi^{(1)} * \varrho_{t_{0}}\right) \frac{d}{d t}\left(\chi^{(2)} * \varrho_{t_{0}}\right)+\left(\chi^{(2)} * \varrho_{t_{0}}\right) \frac{d}{d t}\left(\chi^{(1)} * \varrho_{t_{0}}\right) d y d \eta \\
& =-2 \iint \chi^{(1)}\left(x^{\prime}, \xi^{\prime}, t\right) \varrho_{t_{0}}\left(x^{\prime}, y, \xi^{\prime}, \eta, t\right) \varrho_{t_{0}}(x, y, \xi, \eta, t) \partial_{\xi} m^{(2)}(x, \xi, t) d x d \xi d x^{\prime} d \xi^{\prime} d y d \eta \\
& -2 \iint m^{(1)}\left(x^{\prime}, \xi^{\prime}, t\right) \varrho_{t_{0}}\left(x^{\prime}, y, \xi^{\prime}, \eta, t\right) \varrho_{t_{0}}(x, y, \xi, \eta, t) \partial_{\xi} m^{(1)}(x, \xi, t) d x d \xi d x^{\prime} d \xi^{\prime} d y d \eta \\
& =-2 \iint \chi^{(1)}\left(x^{\prime}, \xi^{\prime}, t\right) \partial_{\xi^{\prime}} \varrho_{t_{0}}\left(x^{\prime}, y, \xi^{\prime}, \eta, t\right) \varrho_{t_{0}}(x, y, \xi, \eta, t) m^{(2)}(x, \xi, t) d x d \xi d x^{\prime} d \xi^{\prime} d y d \eta \\
& -2 \iint \chi^{(2)}\left(x^{\prime}, \xi^{\prime}, t\right) \partial_{\xi^{\prime}} \varrho_{t_{0}}\left(x^{\prime}, y, \xi^{\prime}, \eta, t\right) \varrho_{t_{0}}(x, y, \xi, \eta, t) m^{(1)}(x, \xi, t) d x d \xi d x^{\prime} d \xi^{\prime} d y d \eta \\
& +\operatorname{Err}^{(1,2)}\left(t_{0}, t\right) \\
& =2 \iint\left(\delta\left(\xi^{\prime}\right)-\delta\left(\xi^{\prime}-u^{(1)}\left(x^{\prime}, t\right)\right)\right) \varrho_{t_{0}}\left(x^{\prime}, y, \xi^{\prime}, \eta, t\right) \varrho_{t_{0}}(x, y, \xi, \eta, t) m^{(2)}(x, \xi, t) d x d \xi d x^{\prime} d \xi^{\prime} d y d \eta \\
& +2 \iint\left(\delta\left(\xi^{\prime}\right)-\delta\left(\xi^{\prime}-u^{(2)}\left(x^{\prime}, t\right)\right)\right) \varrho_{t_{0}}\left(x^{\prime}, y, \xi^{\prime}, \eta, t\right) \varrho_{t_{0}}(x, y, \xi, \eta, t) m^{(1)}(x, \xi, t) d x d \xi d x^{\prime} d \xi^{\prime} d y d \eta \\
& +\operatorname{Err}^{(1,2)}\left(t_{0}, t\right) \\
& \leq 2 \iint \varrho_{t_{0}}\left(x^{\prime}, y, 0, \eta, t\right) \varrho_{t_{0}}(x, y, \xi, \eta, t)\left(m^{(2)}+m^{(1)}\right)(x, \xi, t) d x d \xi d x^{\prime} d y d \eta \\
& +\operatorname{Err}^{(1,2)}\left(t_{0}, t\right),
\end{aligned}
$$

where, the inequality is due to the nonnegativity of the Dirac masses $\delta$ and

$$
\left\{\begin{array}{l}
\operatorname{Err}^{(1,2)}\left(t_{0}, t\right):=2 \int\left(\chi^{(1)}\left(x^{\prime}, \xi^{\prime}, t\right) m^{(2)}(x, \xi, t)+\chi^{(2)}\left(x^{\prime}, \xi^{\prime}, t\right) m^{(1)}(x, \xi, t)\right) \\
\left(\varrho_{t_{0}}\left(x^{\prime}, y, \xi^{\prime}, \eta, t\right) \partial_{\xi} \varrho_{t_{0}}(x, y, \xi, \eta, t)+\partial_{\xi^{\prime}} \varrho_{t_{0}}\left(x^{\prime}, y, \xi^{\prime}, \eta, t\right) \varrho_{t_{0}}(x, y, \xi, \eta, t)\right) d y d \eta d x^{\prime} d \xi^{\prime} d x d \xi
\end{array}\right.
$$

Lemma 4.4 below applied to $f(x, \xi, t):=\chi^{(i)}(x, \xi, t)$ and $m:=m^{(i)}$ yields a null $\mathcal{N} \subseteq[0, T]$ with $0 \notin \mathcal{N}$ such that, for every $t_{0} \geq 0$, all $(s, t) \in \Delta([0, T] \backslash \mathcal{N})$ and $i=1,2$,

$$
\begin{aligned}
& 2 \int_{s}^{t} \iint \varrho_{t_{0}}\left(x^{\prime}, y, 0, \eta, r\right) \varrho_{t_{0}}(x, y, \xi, \eta, r) m^{(i)}(x, \xi, r) d x d \xi d x^{\prime} d y d \eta d r \\
& =-\int\left(\operatorname{sgn} * \varrho^{0}\right)(y, \eta)\left[\left(\chi^{(i)} * \varrho_{t_{0}}\right)(y, \eta, t)-\left(\chi^{(i)} * \varrho_{t_{0}}\right)(y, \eta, s)\right] d y d \eta+\int_{s}^{t} \operatorname{Err}^{(i)}\left(t_{0}, r\right) d r,
\end{aligned}
$$

with

$$
\begin{aligned}
\operatorname{Err}^{(i)}\left(t_{0}, t\right)=\int m^{(i)}(x, \xi, t)[ & \int\left(\partial_{\xi^{\prime}} \varrho_{t_{0}}\left(x^{\prime}, y, \xi^{\prime}, \eta, t\right) \varrho_{t_{0}}(x, y, \xi, \eta, t)\right. \\
& \left.\left.+\varrho_{t_{0}}\left(x^{\prime}, y, \xi^{\prime}, \eta, t\right) \partial_{\xi} \varrho_{t_{0}}(x, y, \xi, \eta, t)\right) d y d \eta d x^{\prime} d \xi^{\prime}\right] d x d \xi .
\end{aligned}
$$


It follows that, for all $t_{0} \geq 0$ and all $(s, t) \in \Delta([0, T] \backslash \mathcal{N})$,

$$
\begin{aligned}
& -2 \int\left[\left(\chi^{(1)} * \varrho_{t_{0}}\right)(y, \eta, t)\left(\chi^{(2)} * \varrho_{t_{0}}\right)(y, \eta, t)-\left(\chi^{(1)} * \varrho_{t_{0}}\right)(y, \eta, s)\left(\chi^{(2)} * \varrho_{t_{0}}\right)(y, \eta, s)\right] d y d \eta \\
\leq & -\int\left(\operatorname{sgn} * \varrho^{0}\right)(y, \eta)\left[\left(\chi^{(1)} * \varrho_{t_{0}}\right)(y, \eta, t)-\left(\chi^{(1)} * \varrho_{t_{0}}\right)(y, \eta, s)\right] d y d \eta \\
& -\int\left(\operatorname{sgn} * \varrho^{0}\right)(y, \eta)\left[\left(\chi^{(2)} * \varrho_{t_{0}}\right)(y, \eta, t)-\left(\chi^{(2)} * \varrho_{t_{0}}\right)(y, \eta, s)\right] d y d \eta \\
& +\int_{s}^{t} \operatorname{Err}^{(1)}\left(t_{0}, r\right)+\operatorname{Err}^{(2)}\left(t_{0}, r\right)+\operatorname{Err}^{1,2}\left(t_{0}, r\right) d r .
\end{aligned}
$$

For $t \in[0, T]$ we define

$$
F(t):=\int\left(\chi^{(1)}(y, \eta, t)-\chi^{(2)}(y, \eta, t)\right)^{2} d y d \eta
$$

Let $\varrho_{\varepsilon}^{s}, \varrho_{\varepsilon}^{v}$ be standard smooth approximations of Dirac masses (here we use the superscripts $s$ and $v$ to signify that they are approximations to Dirac masses in space and velocity respectively) and, for each $t_{0} \geq 0$, let $\varrho_{t_{0}, \varepsilon}(x, y, \xi, \eta, t)$ be as in (2.12) with initial condition $\varrho_{\varepsilon}^{0}(x, y, \xi, \eta)=\varrho_{\varepsilon}^{s}(x-y) \varrho_{\varepsilon}^{v}(\xi-\eta)$, that is, for all $t \in[0, T]$,

$$
\varrho_{t_{0}, \varepsilon}(x, y, \xi, \eta, t)=\varrho_{\varepsilon}^{0}\left(\begin{array}{c}
X_{(t, x, \xi)}\left(t-t_{0}\right)-y \\
\Xi_{(t, x, \xi)}\left(t-t_{0}\right)-\eta
\end{array}\right)=\varrho_{\varepsilon}^{s}\left(X_{(t, x, \xi)}\left(t-t_{0}\right)-y\right) \varrho_{\varepsilon}^{v}\left(\Xi_{(t, x, \xi)}\left(t-t_{0}\right)-\eta\right) .
$$

We then define

$$
\begin{aligned}
F_{t_{0}, \varepsilon}(t):= & -2 \int\left(\chi^{(1)} * \varrho_{t_{0}, \varepsilon}\right)(y, \eta, t)\left(\chi^{(2)} * \varrho_{t_{0}, \varepsilon}\right)(y, \eta, t) d y d \eta \\
& +\int \operatorname{sgn}^{\varepsilon}(\eta)\left(\chi^{(1)} * \varrho_{t_{0}, \varepsilon}\right)(y, \eta, t) d y d \eta+\int \operatorname{sgn}^{\varepsilon}(\eta)\left(\chi^{(2)} * \varrho_{t_{0}, \varepsilon}\right)(y, \eta, t) d y d \eta .
\end{aligned}
$$

Lemma 4.5 below, applied to $f(x, \xi, t):=\chi^{(i)}(x, \xi, t)$, implies that, as $\varepsilon \rightarrow 0$ and for all $t_{0} \geq 0$ and all $t \in\left[t_{0}, T\right] \backslash \mathcal{N}$

$$
F_{t_{0}, \varepsilon}(t) \rightarrow-2 \int \chi^{(1)}(y, \eta, t) \chi^{(2)}(y, \eta, t) d y d \eta+\int\left|\chi^{(1)}\right|(y, \eta, t) d y d \eta+\int\left|\chi^{(2)}\right|(y, \eta, t) d y d \eta,
$$

and thus

$$
F_{t_{0}, \varepsilon}(t) \rightarrow F(t)
$$

In view (3.3), for all $t_{0} \geq 0$ and $(s, t) \in \Delta([0, T] \backslash \mathcal{N})$, we find

$$
F_{t_{0}, \varepsilon}(t)-F_{t_{0}, \varepsilon}(s) \leq \int_{s}^{t}\left(\operatorname{Err}^{(1)}\left(t_{0}, r, \varepsilon\right)+\operatorname{Err}^{(2)}\left(t_{0}, r, \varepsilon\right)+\operatorname{Err}^{(1,2)}\left(t_{0}, r, \varepsilon\right)\right) d r
$$

where

$$
\begin{aligned}
& \operatorname{Err}^{(1,2)}\left(t_{0}, r, \varepsilon\right):=2 \int\left[\left(\chi^{(1)}\left(x^{\prime}, \xi^{\prime}, t\right) m^{(2)}(x, \xi, t)+\chi^{(2)}\left(x^{\prime}, \xi^{\prime}, t\right) m^{(1)}(x, \xi, t)\right)\right. \\
& \left.\left(\varrho_{t_{0}, \varepsilon}\left(x^{\prime}, y, \xi^{\prime}, \eta, t\right) \partial_{\xi} \varrho_{t_{0}, \varepsilon}(x, y, \xi, \eta, t)+\partial_{\xi^{\prime}} \varrho_{t_{0}, \varepsilon}\left(x^{\prime}, y, \xi^{\prime}, \eta, t\right) \varrho_{t_{0}, \varepsilon}(x, y, \xi, \eta, t)\right)\right] d y d \eta d x^{\prime} d \xi^{\prime} d x d \xi,
\end{aligned}
$$

and, for $i=1,2$,

$$
\begin{aligned}
\operatorname{Err}^{(i)}\left(t_{0}, r, \varepsilon\right):=\int m^{(i)}(x, \xi, t) & \left(\int \left(\partial_{\xi^{\prime}} \varrho_{t_{0}, \varepsilon}\left(x^{\prime}, y, \xi^{\prime}, \eta, t\right) \varrho_{t_{0}, \varepsilon}(x, y, \xi, \eta, t)\right.\right. \\
& \left.\left.+\varrho_{t_{0}, \varepsilon}\left(x^{\prime}, y, \xi^{\prime}, \eta, t\right) \partial_{\xi} \varrho_{t_{0}, \varepsilon}(x, y, \xi, \eta, t)\right) d y d \eta d x^{\prime} d \xi^{\prime}\right) d x d \xi
\end{aligned}
$$


Lemma B.1 implies that, for all $(s, t) \in \Delta\left(\left[t_{0}, T\right]\right)$ and $i=1,2$,

$$
\begin{aligned}
& \int_{s}^{t} \operatorname{Err}^{(1,2)}\left(t_{0}, r, \varepsilon\right) d r \leq 2\left(\int_{s}^{t} \int m^{(2)}(x, \xi, r)+m^{(1)}(x, \xi, r) d x d \xi d r\right) \\
& \sup _{\substack{(x, \xi) \in \mathbb{R}^{N+1} \\
r \in[s, t]}}\left(\int\left|\int\left(\varrho_{t_{0}, \varepsilon}\left(x^{\prime}, y, \xi^{\prime}, \eta, r\right) \partial_{\xi} \varrho_{t_{0}, \varepsilon}(x, y, \xi, \eta, r)+\partial_{\xi^{\prime}} \varrho_{t_{0}, \varepsilon}\left(x^{\prime}, y, \xi^{\prime}, \eta, r\right) \varrho_{t_{0}, \varepsilon}(x, y, \xi, \eta, r)\right) d y d \eta\right| d x^{\prime} d \xi^{\prime}\right) \\
& \leq C\left(\int_{s}^{t} \int m^{(2)}(x, \xi, r)+m^{(1)}(x, \xi, r) d x d \xi d r\right)\left(t-t_{0}\right)^{\alpha},
\end{aligned}
$$

and, similarly

$$
\int_{s}^{t} \operatorname{Err}^{i}\left(t_{0}, r, \varepsilon\right) \leq C\left(\int_{s}^{t} \int m^{(i)}(x, \xi, r) d x d \xi d r\right)\left(t-t_{0}\right)^{\alpha}
$$

It follows that, for all $(s, t) \in \Delta\left(\left[t_{0}, T\right]\right)$,

$$
\int_{s}^{t} \operatorname{Err}^{1}\left(t_{0}, r, \varepsilon\right)+\operatorname{Err}^{2}\left(t_{0}, r, \varepsilon\right)+\operatorname{Err}^{1,2}\left(t_{0}, r, \varepsilon\right) d r \leq C\left(t-t_{0}\right)^{\alpha} \int_{s}^{t} \int\left(m^{(2)}(x, \xi, r)+m^{(1)}(x, \xi, r)\right) d x d \xi d r .
$$

Next recall that, in view of (3.5), (3.6) and (3.7), there exists a null $\mathcal{N} \subseteq[0, T]$ with $0 \notin \mathcal{N}$ such that for all $(s, t) \in \Delta([0, T] \backslash \mathcal{N})$ and every partition $\Delta=\left\{s=t_{0} \leq \cdots \leq t_{M}=t\right\} \subseteq[0, T] \backslash \mathcal{N}$,

$$
\begin{aligned}
F(t)-F(s) & =\sum_{i=1}^{N} F\left(t_{i+1}\right)-F\left(t_{i}\right) \\
& =\lim _{\varepsilon \rightarrow 0} \sum_{i=1}^{N} F_{t_{i}, \varepsilon}\left(t_{i+1}\right)-F_{t_{i}, \varepsilon}\left(t_{i}\right) \\
& \leq \lim _{\varepsilon \rightarrow 0} \sum_{i=1}^{N} \int_{t_{i}}^{t_{i+1}}\left[E r r^{(1)}\left(t_{i}, r, \varepsilon\right)+\operatorname{Err}^{(2)}\left(t_{i}, r, \varepsilon\right)+E r r^{(1,2)}\left(t_{i}, r, \varepsilon\right)\right] d r \\
& \leq C \sum_{i=1}^{N}\left(t_{i+1}-t_{i}\right)^{\alpha}\left(\int_{t_{i}}^{t_{i+1}} \int\left[m^{(2)}(x, \xi, r)+m^{(1)}(x, \xi, r)\right] d x d \xi d r\right) \\
& \leq C\|\Delta\|^{\alpha}\left(\int_{s}^{t} \int\left[m^{(2)}(x, \xi, r)+m^{(1)}(x, \xi, r)\right] d x d \xi d r\right) .
\end{aligned}
$$

Letting $\|\Delta\| \rightarrow 0$ yields that, for all $(s, t) \in \Delta([0, T] \backslash \mathcal{N}), F(t) \leq F(s)$ and, since, $0 \notin \mathcal{N}$, the claim follows.

\section{The EXISTEnCE of PATHWise ROUGH ENTROPy/KinetiC SOLUtions}

We establish here the existence of pathwise rough entropy/kinetic solutions, which, in view of Theorem 3.1, are unique.

Theorem 4.1. Let $u_{0} \in\left(L^{1} \cap L^{2}\right)\left(\mathbb{R}^{N}\right)$. Then there exists a pathwise rough entropy/kinetic solution $u \in L^{\infty}\left([0, T] ; L^{1}\left(\mathbb{R}^{N}\right)\right)$ to (1.1).

Since the proof is long we first outline the main steps. The solution is found as a weak limit of solutions of problems like (1.1) with regularized driving signals. Due to the lack of BV-type estimates to obtain the necessary compactness we follow the program of Per02] and work with what are known as generalized kinetic solutions. We then show that these solutions converge weakly to a limit that we call a generalized pathwise rough entropy/kinetic solution. The final step is to show that the latter 
is indeed a pathwise rough entropy/kinetic solution to (1.1). Each of the steps described above are discussed in a separate subsection.

The regularized equation and some difficulties. Let $z^{n}$ be a regular approximation of the rough driving signal $z$ (such $z^{n}$ exist since $z$ is assumed to the a geometric rough path - see Appendix A) and consider the approximating kinetic solution $\chi^{n}$ to

$$
\left\{\begin{array}{l}
\partial_{t} \chi^{n}+a^{n}(x, \xi, t) \cdot D_{x} \chi^{n}-b^{n}(x, \xi, t) \partial_{\xi} \chi^{n}=\partial_{\xi} m^{n} \text { in } \mathbb{R}^{N} \times \mathbb{R} \times(0, T), \\
\chi^{n}=\chi\left(u_{0}(\cdot), \cdot\right) \text { on } \mathbb{R}^{N} \times \mathbb{R} \times\{0\}
\end{array}\right.
$$

where

$$
a_{i}^{n}(x, \xi, t):=\sum_{j=1}^{M}\left(\partial_{u} A^{i, j}\right)(x, \xi) \dot{z}^{n, j}(t) \text { and } b^{n}(x, \xi, t):=\sum_{i=1}^{N} \sum_{j=1}^{M}\left(\partial_{x_{i}} A^{i, j}\right)(x, \xi) \dot{z}^{n, j}(t) .
$$

Although not written down the existence of a unique solution $\chi^{n}$ to (4.1) follows from straightforward modifications of standard methods (see, for example, Dal06, Per02]), since $\dot{z}^{n}$ is continuous (actually smooth). In order to pass to the limit $n \rightarrow \infty$ we need to establish some uniform in $n$ estimates on $\chi^{n}$ and $m^{n}$ that do not blow up as $n \rightarrow \infty$. Unfortunately the known estimates do not have this property. For example, the bound on the total mass of the kinetic measure that can be obtained following Dal06, Per02] is

$$
\int_{0}^{T} \int_{\mathbb{R}^{N+1}} m^{n}(x, \xi, t) d x d \xi d t \leq \frac{1}{2}\left\|u^{0}\right\|_{2}^{2}+\int_{0}^{T} \int_{\mathbb{R}^{N+1}}\left|b^{n}(x, \xi, t)\right| d x d \xi d t
$$

and the right hand side blows up as $n \rightarrow \infty$ since it depends on the total variation of $z^{n}$. Following [LPS14] It is therefore necessary to find new bounds on the total mass of $m^{n}$ which is presented in Section 4 .

Once uniform estimates on $\chi^{n}$ and $m^{n}$ have been obtained, we find subsequences, which we denote below the same way as the sequences, which converge in the appropriate weak $\star$ limits in (4.1), that is

$$
\chi^{n} \rightarrow f \text { in } L^{\infty} \text { weak } \star \text { and } m^{n} \rightarrow m \text { in } \mathcal{M} \text { weak } \star .
$$

Although a priori it is unclear whether $f$ is again a characteristic-type function, that is whether $f(x, \xi, t)=\chi(u(t, x), \xi)$ for some $u$, we may pass to the limit in (4.1) to obtain

$$
\left\{\begin{array}{l}
\partial_{t} f+a(x, \xi, t) \cdot D_{x} f-b(x, \xi, t) \partial_{\xi} f=\partial_{\xi} m \text { in } \mathbb{R}^{N} \times \mathbb{R} \times(0, T), \\
f=\chi\left(u_{0}(\cdot), \cdot\right) \text { on } \mathbb{R}^{N} \times \mathbb{R} \times\{0\} .
\end{array}\right.
$$

This observation motivates the concept of generalized pathwise rough entropy/kinetic solutions introduced next.

Generalized pathwise rough entropy/kinetic solutions and some basic properties. We begin with the following definition.

Definition 4.2. Assume that $u_{0} \in\left(L^{1} \cap L^{2}\right)\left(\mathbb{R}^{N}\right)$. Then $f \in L^{\infty}\left([0, T] ; L^{1}\left(\mathbb{R}^{N} \times \mathbb{R}\right)\right) \cap L^{\infty}\left(\mathbb{R}^{N} \times \mathbb{R} \times\right.$ $[0, T])$ is a generalized pathwise rough entropy/kinetic solution to (1.1) if there exists a nonnegative measure $\nu$ and a nonnegative, bounded measure $m$ on $\mathbb{R}^{N} \times \mathbb{R} \times[0, T]$ such that

$$
f(x, \xi, 0)=\chi\left(u_{0}(x), \xi\right),|f|(x, \xi, t)=\operatorname{sgn}(\xi) f(x, \xi, t) \leq 1 \text { and } \frac{\partial f}{\partial \xi}=\delta(\xi)-\nu(x, \xi, t),
$$


and, for all $t_{0} \geq 0$, all test functions $\varrho_{t_{0}}$ given by (2.12) with $\varrho^{0} \in C_{c}^{\infty}\left(\mathbb{R}^{N+1}\right)$ and all $\varphi \in C_{c}^{\infty}([0, T])$,

$$
\int_{0}^{T} \partial_{t} \varphi(r)\left(\varrho_{t_{0}} * f\right)(y, \eta, r) d r+\varphi(0)\left(\varrho_{t_{0}} * f\right)(y, \eta, 0)=\int_{0}^{T} \int \varphi(r) \partial_{\xi} \varrho_{t_{0}}(x, y, \xi, \eta, r) m(x, \xi, t) d x d \xi d r .
$$

It is, of course, immediate that a pathwise rough entropy/kinetic solution is also a generalized one. Moreover, a claim similar to Proposition 2.2 is true here too. Indeed $f$ is a generalized pathwise rough entropy/kinetic solution to (1.1) if and only if there is a null $\mathcal{N} \subseteq[0, T]$ such that $0 \notin \mathcal{N}$ and, for all $t_{0} \geq 0$, all $(s, t) \in \Delta([0, T] \backslash \mathcal{N})$ and all test functions $\varrho_{t_{0}}$ given by (2.12) with $\varrho^{0} \in C_{c}^{\infty}\left(\mathbb{R}^{N+1}\right)$,

$$
\varrho_{t_{0}} * f(y, \eta, t)-\varrho_{t_{0}} * f(y, \eta, s)=-\int_{s}^{t} \int \partial_{\xi} \varrho_{t_{0}}(x, y, \xi, \eta, r) m(x, \xi, r) d x d \xi d r .
$$

We show first that the solutions given by Definition 4.2 are $L^{\infty}\left(\mathbb{R}^{N} \times \mathbb{R}\right)$ weak $\star$ continuous at $t=0$.

Proposition 4.3. Let $f$ be a generalized pathwise rough entropy/kinetic solution to (1.1). Then there exists a null $\mathcal{N} \subseteq[0, T]$ such that, if $t_{n} \in[0, T] \backslash \mathcal{N}$ and $t_{n} \rightarrow 0$ as $n \rightarrow \infty$,

$$
f\left(x, \xi, t_{n}\right) \rightarrow \chi\left(\xi, u_{0}(x)\right) \text { in } L^{\infty}\left(\mathbb{R}^{N} \times \mathbb{R}\right) \text { weak } \star
$$

Proof. The argument is similar to the one in $\left[\right.$ Per02, Proposition 4.1.7]. Since $f \in L^{\infty}\left(\mathbb{R}^{N} \times \mathbb{R} \times[0, T]\right)$ and $m$ is a finite measure on $\mathbb{R}^{N} \times \mathbb{R} \times[0, T]$, there exists a null $\mathcal{N} \subseteq[0, T]$ with the property that, every sequence $t_{n} \in[0, T] \backslash \mathcal{N}$ with $t_{n} \rightarrow 0$ has a subsequence (again denoted by $t_{n}$ ) such that, as $n \rightarrow \infty$,

$$
\begin{array}{r}
f\left(x, \xi, t_{n}\right) \rightarrow F(x, \xi) \text { in } L^{\infty}\left(\mathbb{R}^{N} \times \mathbb{R}\right) \text { weak } \star, \\
\int_{0}^{t_{n}} m(x, \xi, r) d r \rightarrow M(x, \xi) \text { in } \mathcal{M}\left(\mathbb{R}^{N} \times \mathbb{R}\right) \text { weak } \star,
\end{array}
$$

and

$$
\begin{aligned}
& \operatorname{sgn}(\xi) F(x, \xi)=|F|(x, \xi) \leq 1, \\
& M(x, \xi) \geq 0 \text { and } \int M(x, \xi) d x d \xi \leq \int_{0}^{T} \int m(x, \xi, r) d x d \xi d r .
\end{aligned}
$$

Since $f$ is a generalized pathwise rough entropy/kinetic solution we have

$$
\left(\varrho_{0} * f\right)\left(y, \eta, t_{n}\right)-\left(\varrho_{0} * f\right)(y, \eta, 0)=-\int_{0}^{t_{n}} \int \partial_{\xi} \varrho_{0}(x, y, \xi, \eta, r) m(x, \xi, r) d x d \xi d r .
$$

Letting $t_{n} \rightarrow 0$ yields

$$
\left(\varrho^{0} * F\right)(y, \eta)-\left(\varrho^{0} * f^{0}\right)(y, \eta)=-\int \partial_{\xi} \varrho^{0}(x, y, \xi, \eta) M(x, \xi) d x d \xi
$$

and, hence,

$$
F(x, \xi)=\chi\left(\xi, u_{0}(x)\right)+\partial_{\xi} M(x, \xi)
$$

in the sense of distributions. Then [Per02, Lemma 2.2.3] yields $M=0$ and $F(x, \xi, t)=\chi\left(\xi, u_{0}(x)\right)$. 
Two important lemmata. We present here two technical lemmata about the behavior of certain expressions (integrals) involving $f, m$ and the special test functions we are using here. These results were already used in the proof of the contraction proof (uniquess) in the previous section and are also important for the existence. At a first passage the reader may want to skip them and go straight to the next subsection.

Lemma 4.4. Let $f$ be a generalized pathwise rough entropy/kinetic solution. There exists a null $\mathcal{N} \subseteq[0, T]$ with $0 \notin \mathcal{N}$ such that, for every $t_{0} \geq 0$, all $\varrho_{t_{0}}$ as in (2.12) and all $(s, t) \in \Delta([0, T] \backslash \mathcal{N})$,

$$
\begin{aligned}
& 2 \int_{s}^{t} \int \varrho_{t_{0}}\left(x^{\prime}, y, 0, \eta, r\right) \varrho_{t_{0}}(x, y, \xi, \eta, r) m(x, \xi, r) d x d \xi d x^{\prime} d y d \eta d r \\
& =-\int\left(\operatorname{sgn} * \varrho^{0}\right)(y, \eta)\left(f * \varrho_{t_{0}}\right)(y, \eta, t) d y d \eta+\int\left(\operatorname{sgn} * \varrho^{0}\right)(y, \eta)\left(f * \varrho_{t_{0}}\right)(y, \eta, s)+\int_{s}^{t} \operatorname{Err}\left(t_{0}, r\right) d r,
\end{aligned}
$$

where

$$
\begin{aligned}
\operatorname{Err}\left(t_{0}, t\right)=-\int m(x, \xi, t) \operatorname{sgn}\left(\xi^{\prime}\right) & \left(\partial_{\xi^{\prime}} \varrho_{t_{0}}\left(x^{\prime}, y, \xi^{\prime}, \eta, t\right) \varrho_{t_{0}}(x, y, \xi, \eta, t)\right. \\
& \left.+\varrho_{t_{0}}\left(x^{\prime}, y, \xi^{\prime}, \eta, t\right) \partial_{\xi} \varrho_{t_{0}}(x, y, \xi, \eta, t)\right) d y d \eta d x^{\prime} d \xi^{\prime} d x d \xi .
\end{aligned}
$$

Proof. For all $s \leq t$ we have

$$
\begin{aligned}
& 2 \int_{s}^{t} \int \varrho_{t_{0}}\left(x^{\prime}, y, 0, \eta, r\right) \varrho_{t_{0}}(x, y, \xi, \eta, r) m(x, \xi, r) d x d \xi d x^{\prime} d y d \eta d r \\
& =\int_{s}^{t} \int \partial_{\xi^{\prime}} \operatorname{sgn}\left(\xi^{\prime}\right) \varrho_{t_{0}}\left(x^{\prime}, y, \xi^{\prime}, \eta, r\right) \varrho_{t_{0}}(x, y, \xi, \eta, r) m(x, \xi, r) d x d \xi d x^{\prime} d \xi^{\prime} d y d \eta d r \\
& =\int_{s}^{t} \int\left(\int \operatorname{sgn}\left(\xi^{\prime}\right) \varrho_{t_{0}}\left(x^{\prime}, y, \xi^{\prime}, \eta, r\right) d x^{\prime} d \xi^{\prime}\right)\left(\int \partial_{\xi} \varrho_{t_{0}}(x, y, \xi, \eta, r) m(x, \xi, r) d x d \xi\right) d y d \eta d r \\
& +\int_{s}^{t} \operatorname{Err}\left(t_{0}, r\right) d r .
\end{aligned}
$$

It follows from (2.11) that

$$
\begin{aligned}
& \int \operatorname{sgn}\left(\xi^{\prime}\right) \varrho_{t_{0}}\left(x^{\prime}, y, \xi^{\prime}, \eta, t\right) d x^{\prime} d \xi^{\prime} \\
& =\int \operatorname{sgn}\left(\Xi_{\left(t, x^{\prime}, \xi^{\prime}\right)}\left(t-t_{0}\right)\right) \varrho^{0}\left(\begin{array}{c}
X_{\left(t, x^{\prime}, \xi^{\prime}\right)}\left(t-t_{0}\right)-y \\
\Xi_{\left(t, x^{\prime}, \xi^{\prime}\right)}\left(t-t_{0}\right)-\eta
\end{array}\right) d x^{\prime} d \xi^{\prime} \\
& =\int \operatorname{sgn}\left(\xi^{\prime}\right) \varrho^{0}\left(\begin{array}{c}
x^{\prime}-y \\
\xi^{\prime}-\eta
\end{array}\right) d x^{\prime} d \xi^{\prime} \\
& =\left(\operatorname{sgn} * \varrho^{0}\right)(y, \eta) .
\end{aligned}
$$

Hence, there exists a null $\mathcal{N} \subseteq[0, T]$ with $0 \notin \mathcal{N}$ such that, for every $t_{0} \geq 0$ and all $(s, t) \in$ $\Delta\left(\left[t_{0}, T\right] \backslash \mathcal{N}\right)$

$$
\begin{aligned}
& 2 \int_{s}^{t} \int \varrho_{t_{0}}\left(x^{\prime}, y, 0, \eta, r\right) \varrho_{t_{0}}(x, y, \xi, \eta, r) m(x, \xi, r) d x d \xi d x^{\prime} d \xi^{\prime} d y d \eta d r \\
& =\int_{s}^{t} \int\left(\operatorname{sgn} * \varrho^{0}\right)(y, \eta)\left(\int \partial_{\xi} \varrho_{t_{0}}(x, y, \xi, \eta, r) m(x, \xi, r) d x d \xi\right) d y d \eta+\int_{s}^{t} \operatorname{Err}\left(t_{0}, r\right) d r \\
& =-\left.\int\left(\operatorname{sgn} * \varrho^{0}\right)(y, \eta)\left(f * \varrho_{t_{0}}\right)(y, \eta, \cdot)\right|_{s} ^{t} d y d \eta+\int_{s}^{t} \operatorname{Err}\left(t_{0}, r\right) d r .
\end{aligned}
$$


As in the proof of Theorem 3.1 let $\varrho_{\varepsilon}^{s}, \varrho_{\varepsilon}^{v}$ be standard smooth approximations of Dirac masses and, for each $t_{0} \geq 0$, let $\varrho_{t_{0}, \varepsilon}(x, y, \xi, \eta, t)$ be as in (2.12) with initial condition $\varrho_{\varepsilon}^{0}(x, y, \xi, \eta)=\varrho_{\varepsilon}^{s}(x-y) \varrho_{\varepsilon}^{v}(\xi-\eta)$, that is $\varrho_{t_{0}, \varepsilon}$ is as in (3.4).

Lemma 4.5. Let $f$ be a generalized pathwise rough entropy/kinetic solution to (1.1). There exists a null $\mathcal{N} \subseteq[0, T]$ with $0 \notin \mathcal{N}$ such that, for every $t_{0} \geq 0$ and all $t \in\left[t_{0}, T\right] \backslash \mathcal{N}$, as $\varepsilon \rightarrow 0$

$$
f * \varrho_{t_{0}, \varepsilon}(y, \eta, t) \rightarrow f\left(Y_{\left(t_{0}, y, \eta\right)}(t), \zeta_{\left(t_{0}, y, \eta\right)}(t), t\right),
$$

where $\varrho_{t_{0}, \varepsilon}$ is as in (3.4).

Proof. The proof is immediate, since, for a.e. $t \in\left[t_{0}, T\right]$, as $\varepsilon \rightarrow 0$,

$$
\begin{aligned}
f * \varrho_{t_{0}, \varepsilon}(y, \eta, t) & =\int f(x, \xi, t) \varrho_{t_{0}, \varepsilon}(x, y, \xi, \eta, t) d x d \xi \\
& =\int f(x, \xi, t) \varrho_{\varepsilon}^{s}\left(X_{(t, x, \xi)}\left(t-t_{0}\right)-y\right) \varrho_{\varepsilon}^{v}\left(\Xi_{(t, x, \xi)}\left(t-t_{0}\right)-\eta\right) d x d \xi \\
& =\int f\left(Y_{\left(t_{0}, x, \xi\right)}(t), \zeta_{\left(t_{0}, x, \xi\right)}(t), t\right) \varrho_{\varepsilon}^{s}(x-y) \varrho_{\varepsilon}^{s}(\xi-\eta) d x d \xi \\
& \rightarrow f\left(Y_{\left(t_{0}, y, \eta\right)}(t), \zeta_{\left(t_{0}, y, \eta\right)}(t), t\right),
\end{aligned}
$$

and, in light of $f(x, \xi, 0)=\chi\left(\xi, u_{0}(x)\right)$, this is true, in particular, for $t=0$.

Stable a priori estimates. We use here the main idea of the definition of the pathwise rough entropy/kinetic solution, namely the use of test functions that propagate along the characteristics of the kinetic equation, to improve (4.2).

We begin with the following preliminary result.

Lemma 4.6. Let $f$ be a generalized pathwise rough entropy/kinetic solution to (1.1). There exists a null $\mathcal{N} \subseteq[0, T]$ such that, for all $t \in[0, T] \backslash \mathcal{N}$,

$$
\int|f|(x, \xi, t) d x d \xi \leq\left\|u_{0}\right\|_{1} .
$$

Proof. Fix $\varphi \in C_{c}^{\infty}(\mathbb{R})$ monotone with $\varphi(0)=0$ and use $\varrho_{t_{0}}=\varphi\left(\Xi_{(t, x, \xi)}\left(t-t_{0}\right)\right)$ as a test function with $(y, \eta)=(0,0)$ to get a null $\mathcal{N} \subseteq[0, T]$ such that $0 \notin \mathcal{N}$ and, for all $t_{0} \in[0, T],(s, t) \in \Delta\left(\left[t_{0}, T\right] \backslash \mathcal{N}\right)$,

$$
\begin{aligned}
\int f(x, \xi, t) \varphi\left(\Xi_{(t, x, \xi)}\left(t-t_{0}\right)\right) d x d \xi- & \int f(x, \xi, s) \varphi\left(\Xi_{(s, x, \xi)}\left(s-t_{0}\right)\right) d x d \xi \\
= & -\int_{s}^{t} \int \partial_{\xi} \varphi\left(\Xi_{(r, x, \xi)}\left(r-t_{0}\right)\right) m(x, \xi, r) d x d \xi d r .
\end{aligned}
$$

Choosing $s=t_{0} \notin \mathcal{N}$ we find

$$
\begin{aligned}
\int_{s}^{t} \int \partial_{\xi} \varphi\left(\Xi_{(r, x, \xi)}(r-s)\right) \partial_{\xi} \Xi_{(r, x, \xi)}(r-s) m(x, \xi, r) d \xi d x d r \\
+\int f(x, \xi, t) \varphi\left(\Xi_{(t, x, \xi)}(t-s)\right) d x d \xi \leq \int f(x, \xi, s) \varphi(\xi) d x d \xi
\end{aligned}
$$

Since

$$
\operatorname{sgn}\left(\Xi_{(t, x, \xi)}(t-s)\right)=\operatorname{sgn}(f(x, \xi, t))=\operatorname{sgn}(\xi),
$$

it follows that, for all $t \in\left[t_{0}, T\right] \backslash \mathcal{N}$ and a.e. in $x$ and $\xi$,

$$
f(x, \xi, t) \varphi\left(\Xi_{(t, x, \xi)}\left(t-t_{0}\right)\right) \geq 0 .
$$


In view of Lemma A.1, we know that there exists $C=C(R)>0$ such that, for all $\alpha$-Hölder rough paths $z$ with $\|z\|_{\alpha-\mathrm{Höl} ;[0, T]} \leq R$ and all $r \in[0, t]$,

$$
\left|\partial_{\xi} \Xi_{(r, x, \xi)}(t)-1\right|=\left|\partial_{\xi} \Xi_{(r, x, \xi)}(t)-\partial_{\xi} \Xi_{(r, x, \xi)}(0)\right| \leq\left\|\partial_{\xi} \Xi_{(r, x, \xi)}(t)\right\|_{\alpha-\mathrm{Höl} ;[0, r]}|t|^{\alpha} \leq C|t|^{\alpha} .
$$

Hence, for $h>0$ small enough and all $|t-s| \leq h$, we have

$$
\inf _{r \in[s, t]} \partial_{\xi} \Xi_{(r, x, \xi)}(r-s) \geq 0
$$

and, hence,

$$
\int_{s}^{t} \int \partial_{\xi} \varphi \Xi_{(r, x, \xi)}(r-s)\left(\partial_{\xi} \Xi_{(r, x, \xi)}(r-s) m(x, \xi, r) d \xi d x d r \geq 0,\right.
$$

and, from (4.5), for all $s, t \in \Delta([0, T] \backslash \mathcal{N})$ with $|t-s| \leq h$,

$$
\int f(x, \xi, t) \varphi\left(\Xi_{(t, x, \xi)}(t-s)\right) d x d \xi \leq \int f(x, \xi, s) \varphi(\xi) d x d \xi
$$

We now extend the previous estimate to $\varphi: \mathbb{R} \rightarrow \mathbb{R}$ which are measurable and monotone with $\varphi(0)=0$. Given such $\varphi$ let $\varphi^{n} \in C_{c}^{\infty}(\mathbb{R})$ be monotone functions such that $\varphi^{n}(0)=0$ and $\varphi^{n} \rightarrow \varphi$ pointwise. It follows from Fatou's Lemma that

$$
\int f(x, \xi, t) \varphi\left(\Xi_{(t, x, \xi)}(t-s)\right) d x d \xi \leq \liminf _{n \rightarrow \infty} \int f(x, \xi, s) \varphi^{n}(\xi) d x d \xi
$$

Next observe that we may choose $M=M(R)>0$, uniformly for all $\alpha$-Hölder rough paths $z$ with $\|z\|_{\alpha-\mathrm{Höl} ;[0, T]} \leq R$ and, in view of (4.6), for each $t \in[0, T] \backslash \mathcal{N}$, a partition $\Delta=\left\{0=t_{0} \leq \cdots \leq t_{M}=\right.$ $t\} \subseteq[0, T] \backslash \mathcal{N}$ such that, for all $i=0, \ldots, M-1$,

$$
\inf _{r \in\left[t_{i}, t_{i+1}\right]} \partial_{\xi} \Xi_{(r, x, \xi)}(r-s) \geq 0 .
$$

The claim now follows from an elementary iterative argument: Choosing $\varphi=\operatorname{sgn}, \varphi^{n}$ such that $\left|\varphi^{n}\right| \leq|\varphi|$ and $(s, t)=\left(0, t_{1}\right)$ in (4.7) we find

$$
\int f\left(x, \xi, t_{1}\right) \operatorname{sgn}\left(\Xi_{\left(t_{1}, x, \xi\right)}\left(t_{1}\right)\right) d x d \xi \leq \int f(x, \xi, 0) \operatorname{sgn}(\xi) d x d \xi=\int|f|(x, \xi, 0) d x d \xi=\left\|u_{0}\right\|_{1}^{1},
$$

and, since $\operatorname{sgn}\left(\Xi_{\left(t_{1}, x, \xi\right)}\left(t_{1}\right)\right)=\operatorname{sgn}(\xi)$,

$$
\int|f|\left(x, \xi, t_{1}\right) d x d \xi \leq \int|f|(x, \xi, 0) d x d \xi=\left\|u_{0}\right\|_{1}^{1}
$$

Iterating this argument over $i$ yields, for all $t \in[0, T] \backslash \mathcal{N}$,

$$
\int|f|(x, \xi, t) d x d \xi \leq\left\|u_{0}\right\|_{1}^{1} .
$$

We present now the estimate on the total mass which is new even in the class of homogeneous conservation laws without rough time dependence.

Lemma 4.7. Let $f$ be a generalized pathwise rough entropy/kinetic solution to (1.1). There exists a null $\mathcal{N} \subseteq[0, T]$ and, for each $R>0, M=M(R)>0$ such that, for all driving signals z with $\|z\|_{\alpha-\mathrm{Höl} ;[0, T]} \leq R$ and all $t \in[0, T] \backslash \mathcal{N}$,

$$
\frac{1}{2} \int_{0}^{t} \int m(x, \xi, r) d \xi d x d r+\int f(x, \xi, t) \xi d x d \xi \leq \frac{1}{2}\left\|u_{0}\right\|_{2}^{2}+M\left\|u_{0}\right\|_{1} .
$$


Proof. In view of (4.5), for $\varphi \in C_{c}^{\infty}(\mathbb{R})$ monotone with $\varphi(0)=0$, a null set $\mathcal{N} \subseteq[0, T]$ with $0 \notin \mathcal{N}$ and for all $(s, t) \in \Delta([0, T] \backslash \mathcal{N})$, we have

$$
\begin{aligned}
\int_{s}^{t} \int \partial_{\xi} \varphi\left(\Xi_{(r, x, \xi)}(r\right. & -s)) \partial_{\xi} \Xi_{(r, x, \xi)}(r-s) m(x, \xi, r) d \xi d x d r \\
& +\int f(x, \xi, t) \varphi\left(\Xi_{(t, x, \xi)}(t-s)\right) d x d \xi \leq \int f(x, \xi, s) \varphi(\xi) d x d \xi .
\end{aligned}
$$

Moreover, in light of (4.6), for $h>0$ small enough and all for all $|t-s| \leq h$, we have

$$
\inf _{r \in[s, t]} \partial_{\xi} \Xi_{(r, x, \xi)}(r-s) \geq \frac{1}{2}
$$

Hence, for all $s, t \in \Delta([0, T] \backslash \mathcal{N})$ with $|t-s| \leq h$,

$$
\begin{aligned}
\frac{1}{2} \int_{s}^{t} \int & \partial_{\xi} \varphi\left(\Xi_{(r, x, \xi)}(r-s)\right) m(x, \xi, r) d \xi d x d r \\
& +\int f(x, \xi, t) \varphi\left(\Xi_{(t, x, \xi)}(t-s)\right) d x d \xi \leq \int f(x, \xi, s) \varphi(\xi) d x d \xi
\end{aligned}
$$

Let $\varphi \in C^{1}(\mathbb{R})$ be monotone with $\varphi(0)=0$ and choose $\varphi^{n} \in C_{c}^{\infty}(\mathbb{R})$ monotone with $\varphi^{n}(0)=0$ and such that $\varphi^{n} \rightarrow \varphi$ and $\partial_{\xi} \varphi^{n} \rightarrow \partial_{\xi} \varphi$ pointwise. Again Fatou's Lemma yields, for all $s, t \in \Delta([0, T] \backslash \mathcal{N})$ with $|t-s| \leq h$,

$$
\begin{aligned}
\frac{1}{2} \int_{s}^{t} \int & \left(\partial_{\xi} \varphi\right)\left(\Xi_{(r, x, \xi)}(r-s)\right) m(x, \xi, r) d \xi d x d r \\
\quad+\int f(x, \xi, t) \varphi\left(\Xi_{(t, x, \xi)}(t-s)\right) d x d \xi & \leq \liminf _{n \rightarrow \infty} \int f(x, \xi, s) \varphi^{n}(\xi) d x d \xi
\end{aligned}
$$

Letting $\varphi(\xi)=\xi$ in the inequality above gives

$$
\frac{1}{2} \int_{s}^{t} \int m(x, \xi, r) d \xi d x d r+\int f(x, \xi, t) \Xi_{(t, x, \xi)}(t-s) d x d \xi \leq \int f(x, \xi, s) \xi d x d \xi .
$$

Once again it follows from Lemma A.1 that, for each $R>0$, there exists $C=C(R)>0$ such that, for all $\alpha$-Hölder rough paths $z$ with $\|z\|_{\alpha-\mathrm{Hö} ; ;[0, T]} \leq R$ and all $r \in[0, t]$,

$$
\left|\Xi_{(r, x, \xi)}(t)-\xi\right|=\left|\Xi_{(r, x, \xi)}(t)-\Xi_{(r, x, \xi)}(0)\right| \leq\left\|\Xi_{(r, x, \xi)}(t)\right\|_{\alpha-\mathrm{Höl} ;[0, r]}|t|^{\alpha} \leq C|t|^{\alpha} .
$$

In light of (4.6) and (4.8), for each $R>0$ we can find $M=M(R)>0$ such that, for all $\alpha$ Hölder rough paths $z$ with $\|z\|_{\alpha-\text { Höl; }[0, T]} \leq R$ and for each $t \in[0, T] \backslash \mathcal{N}$, there exists a partition $\Delta=\left\{0=t_{0} \leq \cdots \leq t_{M}=t\right\} \subseteq[0, T] \backslash \mathcal{N}$ such that $\|\Delta\| \leq 1$ and, for all $i=0, \ldots, M-1$

$$
\inf _{r \in\left[t_{i}, t_{i+1}\right]} \partial_{\xi} \Xi_{(r, x, \xi)}\left(r-t_{i}\right) \geq \frac{1}{2} \quad \text { and } \sup _{r \in\left[t_{i}, t_{i+1}\right]}\left|\Xi_{(r, x, \xi)}\left(r-t_{i}\right)-\xi\right| \leq 1 .
$$

Observe that

$$
\frac{1}{2} \int_{0}^{t_{1}} \int m(x, \xi, r) d \xi d x d r+\int f\left(x, \xi, t_{1}\right) \Xi_{\left(t_{1}, x, \xi\right)}\left(t_{1}\right) d x d \xi \leq \int f(x, \xi, 0) \xi d x d \xi=\frac{1}{2}\left\|u_{0}\right\|_{2}^{2}
$$


Moreover, using Lemma 4.6, we obtain

$$
\begin{aligned}
\int f\left(x, \xi, t_{1}\right) \xi d x d \xi & =\int f\left(x, \xi, t_{1}\right) \Xi_{\left(t_{1}, x, \xi\right)}\left(t_{1}\right) d x d \xi+\int f\left(x, \xi, t_{1}\right)\left(\xi-\Xi_{\left(t_{1}, x, \xi\right)}\left(t_{1}\right)\right) d x d \xi \\
& \leq \int f\left(x, \xi, t_{1}\right) \Xi_{\left(t_{1}, x, \xi\right)}\left(t_{1}\right) d x d \xi+\int|f|\left(x, \xi, t_{1}\right) d x d \xi \\
& \leq \int f\left(x, \xi, t_{1}\right) \Xi_{\left(t_{1}, x, \xi\right)}\left(t_{1}\right) d x d \xi+\left\|u_{0}\right\|_{1} .
\end{aligned}
$$

Thus

$$
\frac{1}{2} \int_{0}^{t_{1}} \int m(x, \xi, r) d \xi d x d r+\int f\left(x, \xi, t_{1}\right) \xi d x d \xi \leq \int f(x, \xi, 0) \xi d x d \xi+\left\|u_{0}\right\|_{1} .
$$

Iterating the above argument over $i$ yields

$$
\begin{aligned}
\frac{1}{2} \int_{0}^{t} \int m(x, \xi, r) d \xi d x d r+\int f(x, \xi, t) \xi d x d \xi & \leq \int f(x, \xi, 0) \xi d x d \xi+M\left\|u_{0}\right\|_{1} \\
& =\frac{1}{2}\left\|u_{0}\right\|_{2}^{2}+M\left\|u_{0}\right\|_{1} .
\end{aligned}
$$

The construction of generalized pathwise rough entropy/kinetic solutions. The following theorem asserts the existence of the generalized pathwise rough entropy/kinetic solutions.

Theorem 4.8. For each $u_{0} \in\left(L^{1} \cap L^{2}\right)\left(\mathbb{R}^{N}\right)$ there exists a generalized pathwise rough entropy/kinetic solution $f \in L^{\infty}\left([0, T] ; L^{1}\left(\mathbb{R}^{N} \times \mathbb{R}\right)\right) \cap L^{\infty}\left(\mathbb{R}^{N} \times \mathbb{R} \times[0, T]\right)$ to (1.1).

Proof. Given a geometric rough path $z$ choose smooth $z^{n}$ such that $z^{n} \rightarrow z$ in the rough paths metric, that is in $C_{0}^{0, \beta-\mathrm{Höl}}\left([0, T], G^{\lfloor 1 / \beta\rfloor}\left(\mathbb{R}^{N}\right)\right)$, and consider the unique kinetic solutions $\left(u^{n}, m^{n}\right)$ to (1.1), that is $\chi^{n}=\chi\left(u^{n}\right)$ solving (4.1).

We use Lemma 2.3 with $t_{0} \geq 0$ and test functions $\varrho_{t_{0}}^{n}$ given by (2.8) (with $z$ replaced by $z^{n}$ ) and $\varrho^{0} \in C_{c}^{\infty}\left(\mathbb{R}^{N+1}\right)$ to find, for all $\varphi \in C_{c}^{\infty}([0, T))$,

$$
\begin{aligned}
\int_{0}^{T} \partial_{t} \varphi(r)\left(\varrho_{t_{0}}^{n} * \chi^{n}\right)(y, \eta, r) d r+ & \varphi(0)\left(\varrho_{t_{0}}^{n} * \chi^{n}\right)(y, \eta, 0) \\
& =\int_{0}^{T} \int \varphi(r) \partial_{\xi} \varrho_{t_{0}}^{n}(x, y, \xi, \eta, r) m^{n}(x, \xi, r) d x d \xi d r
\end{aligned}
$$

and, hence,

$$
\begin{aligned}
& \int_{0}^{T} \int \partial_{t} \varphi(r) \varrho_{t_{0}}^{n}(x, y, \xi, \eta, r) \chi^{n}(x, \xi, r) d x d \xi d r+\int \varphi(0) \varrho_{t_{0}}^{n}(x, y, \xi, \eta, 0) \chi\left(u_{0}(x), \xi\right) d x d \xi \\
& =\int_{0}^{T} \int \varphi(r) \partial_{\xi} \varrho_{t_{0}}^{n}(x, y, \xi, \eta, r) m^{n}(x, \xi, r) d x d \xi d r .
\end{aligned}
$$

Since, for all $t \in[0, T]$,

$$
\varrho_{t_{0}}^{n}(x, y, \xi, \eta, t)=\varrho^{0}\left(\begin{array}{c}
X_{(t, x, \xi)}^{n}\left(t-t_{0}\right)-y \\
\Xi_{(t, x, \xi)}^{n}\left(t-t_{0}\right)-\eta
\end{array}\right),
$$

and, as $n \rightarrow \infty$, (see Appendix $\mathrm{A}$ )

$$
\sup _{x, \xi}\left\|\left(\begin{array}{c}
X_{(\cdot, x, \xi)}^{n}\left(\cdot-t_{0}\right) \\
\Xi_{(\cdot, x, \xi)}^{n}\left(\cdot-t_{0}\right)
\end{array}\right)-\left(\begin{array}{c}
X_{(\cdot, x, \xi)}\left(\cdot-t_{0}\right) \\
\Xi_{(\cdot, x, \xi)}\left(\cdot-t_{0}\right)
\end{array}\right)\right\|_{C^{0}([0, T])} \rightarrow 0
$$


we have, as $n \rightarrow \infty$ and uniformly in $(x, \xi, t)$,

$$
\varrho_{t_{0}}^{n}(x, y, \xi, \eta, t) \rightarrow \varrho_{t_{0}}(x, y, \xi, \eta, t)
$$

Moreover, standard rough path estimates (see Appendix $\mathrm{A}$ ) yield $C>0$ such that

$$
\sup _{x, \xi}\left\|\left(\begin{array}{c}
X_{(\cdot, x, \xi)}^{n}\left(\cdot-t_{0}\right)-x \\
\Xi_{(\cdot, x, \xi)}^{n}\left(\cdot-t_{0}\right)-\xi
\end{array}\right)\right\|_{C^{0}([0, T])} \leq C .
$$

An immediate consequence of (4.11) and the fact that $\varrho^{0}$ has compact support is that, for each $(y, \eta)$, the $\varrho_{t_{0}}^{n}$ 's also have uniform in $n$ compact support, that is there exists a compact $K \subseteq \mathbb{R}^{N} \times \mathbb{R}$ such that, for all $(x, \xi) \notin K$,

In addition Lemma 4.6 yields

$$
\varrho_{t_{0}}^{n}(x, y, \xi, \eta, t)=0
$$

$$
\sup _{t \in[0, T] \backslash \mathcal{N}}\left\|\chi^{n}(\cdot, \cdot, t)\right\|_{L^{1}\left(\mathbb{R}^{N} \times \mathbb{R}\right)} \leq\left\|u_{0}\right\|_{1} .
$$

Using $\left|\chi^{n}\right| \leq 1$ this implies that, along a subsequence, which for simplicity is denoted by $\chi^{n}$,

$$
\chi^{n} \rightarrow f \quad \text { in } L^{\infty}\left(\mathbb{R}^{N} \times \mathbb{R} \times[0, T]\right) \text { weak } \star \text { and }\|f\|_{L^{\infty}\left([0, T] ; L^{1}\left(\mathbb{R}^{N} \times \mathbb{R}\right)\right)} \leq\left\|u_{0}\right\|_{1} .
$$

It then follows from Per02, Lemma 2.3.1] that there exists a non-negative measure $\nu$ such that

$$
|f|(x, \xi, t)=\operatorname{sgn}(\xi) f(x, \xi, t) \leq 1 \text { and } \partial_{\xi} f(x, \xi, t)=\delta(\xi)-\nu(t, x, \xi) .
$$

Next we use Lemma 4.7, with $\int \chi^{n}(x, \xi, t) \xi d x d \xi=\frac{1}{2}\left\|u^{n}(t)\right\|_{2}^{2}$, to get, for all $t \in[0, T] \backslash \mathcal{N}$,

$$
\frac{1}{2} \int_{0}^{t} \int m^{n}(x, \xi, r) d \xi d x d r+\frac{1}{2}\left\|u^{n}(t)\right\|_{2}^{2} \leq \frac{1}{2}\left\|u_{0}\right\|_{2}^{2}+M\left\|u_{0}\right\|_{1} ;
$$

note that $M$ may be chosen uniformly in $n$ since $\left\|z^{n}\right\|_{\alpha-\mathrm{Höl} ;[0, T]} \leq R$ uniformly for some $R>0$.

It follows that there exists a weak $\star$ convergent subsequence $m^{n} \rightarrow m$ and taking the limit in (4.10) yields

$$
\begin{aligned}
& \int_{0}^{T} \int \partial_{t} \varphi(r) \varrho_{t_{0}}(x, y, \xi, \eta, r) f(x, \xi, r) d x d \xi d r+\int \varphi(0) \varrho_{t_{0}}(x, y, \xi, \eta, 0) \chi\left(u_{0}(x), \xi\right) d x d \xi \\
& =\int_{0}^{T} \int \varphi(r) \partial_{\xi} \varrho_{t_{0}}(x, y, \xi, \eta, r) m(x, \xi, r) d x d \xi d r .
\end{aligned}
$$

Hence, $f$ is a generalized pathwise rough entropy/kinetic solution to (1.1).

The reconstruction of a pathwise rough entropy/kinetic solution from a generalized one. We show here how to go from generalized to pathwise rough entropy/kinetic solution.

Proposition 4.9. Let $f$ be a generalized pathwise rough entropy/kinetic solution to (1.1). There exists $u \in L^{\infty}\left([0, T] ; L^{1}\left(\mathbb{R}^{N}\right)\right)$ such that, a.e. in $(x, \xi, t), f(x, \xi, t)=\chi(\xi, u(x, t))$.

Proof. The claim follows, if we show that, for a given generalized pathwise rough entropy/kinetic solution, there exists a null set $\mathcal{N} \subseteq[0, T]$ with $0 \notin \mathcal{N}$ such that, for all $t \in[0, T] \backslash \mathcal{N}$,

$$
\int\left(f^{2}(y, \eta, t)-|f|(y, \eta, t)\right) d y d \eta=0,
$$

which, in view of (4.3), implies that $f=\chi(u)$ for some measurable $u:[0, T] \times \mathbb{R}^{N} \rightarrow \mathbb{R}$. Since

$$
\|u(\cdot, t)\|_{L^{1}\left(\mathbb{R}^{N}\right)}=\int|\chi|(\xi, u(x, t)) d \xi d x=\int|f|(x, \xi, t) d \xi d x=\|f(\cdot, t)\|_{L^{1}\left(\mathbb{R}^{N} \times \mathbb{R}\right)},
$$


it then follows that $u \in L^{\infty}\left([0, T] ; L^{1}\left(\mathbb{R}^{N}\right)\right)$.

In view of the obvious inequality $\int\left(f^{2}(y, \eta, t)-|f|(y, \eta, t)\right) d y d \eta \leq 0$, to prove (4.12) we have to show that

$$
\int\left(f^{2}(y, \eta, t)-|f|(y, \eta, t)\right) d y d \eta \geq 0
$$

Let $\varrho_{t_{0}, \varepsilon}$ be as in (3.4). From Lemma 4.5 we know that there exists a null $\mathcal{N} \subseteq[0, T]$ with $0 \notin \mathcal{N}$ such that, for all $t \in[0, T] \backslash \mathcal{N}$, as $\varepsilon \rightarrow 0$,

$$
f * \varrho_{t_{0}, \varepsilon}(y, \eta, t) \rightarrow f\left(Y_{\left(t_{0}, y, \eta\right)}(t), \zeta_{\left(t_{0}, y, \eta\right)}(t), t\right),
$$

and, hence, for all $t \in[0, T] \backslash \mathcal{N}$,

$$
\begin{aligned}
\int\left(f^{2}(y, \eta, t)-|f|(y, \eta, t)\right) d y d \eta & =\int f^{2}\left(Y_{\left(t_{0}, y, \eta\right)}(t), \zeta_{\left(t_{0}, y, \eta\right)}(t), t\right) d y d \eta-\int \operatorname{sgn}(\eta) f(y, \eta, t) d y d \eta \\
& =\lim _{\varepsilon \rightarrow 0} \int\left(f * \varrho_{t_{0}, \varepsilon}\right)^{2}(y, \eta, t) d y d \eta-\int \operatorname{sgn}(\eta) f(y, \eta, t) d y d \eta .
\end{aligned}
$$

Thus to show (4.13) it suffices to obtain an appropriate lower bound for

$$
\int\left(f * \varrho_{t_{0}, \varepsilon}\right)^{2}(y, \eta, t) d y d \eta-\int \operatorname{sgn}(\eta) f(y, \eta, t) d y d \eta .
$$

To estimate the first term we note, that, for $t \in\left(t_{0}, T\right)$,

$$
\begin{aligned}
\partial_{t} \int\left(f * \varrho_{t_{0}, \varepsilon}\right)^{2} d y d \eta= & 2 \int\left(f * \varrho_{t_{0}, \varepsilon}\right) \partial_{t}\left(f * \varrho_{t_{0}, \varepsilon}\right) d y d \eta \\
= & -2 \int\left(f * \varrho_{t_{0}, \varepsilon}\right)(y, \eta, t) \partial_{\xi} \varrho_{t_{0}, \varepsilon}(x, y, \xi, \eta, t) m(x, \xi, t) d x d \xi d y d \eta \\
= & -2 \int f\left(x^{\prime}, \xi^{\prime}, t\right) \varrho_{t_{0}, \varepsilon}\left(x^{\prime}, y, \xi^{\prime}, \eta, t\right) \partial_{\xi} \varrho_{t_{0}, \varepsilon}(x, y, \xi, \eta, t) m(x, \xi, t) d x^{\prime} d \xi^{\prime} d x d \xi d y d \eta \\
= & 2 \int f\left(x^{\prime}, \xi^{\prime}, t\right) \partial_{\xi^{\prime}} \varrho_{t_{0}, \varepsilon}\left(x^{\prime}, y, \xi^{\prime}, \eta, t\right) \varrho_{t_{0}, \varepsilon}(x, y, \xi, \eta, t) m(x, \xi, t) d x^{\prime} d \xi^{\prime} d x d \xi d y d \eta \\
& +\operatorname{Err}^{(1)}\left(t_{0}, t, \varepsilon\right),
\end{aligned}
$$

where

$$
\begin{aligned}
\operatorname{Err}^{(1)}\left(t_{0}, t, \varepsilon\right)= & -2 \int f\left(x^{\prime}, \xi^{\prime}, t\right) m(x, \xi, t)\left[\varrho_{t_{0}, \varepsilon}\left(x^{\prime}, y, \xi^{\prime}, \eta, t\right) \partial_{\xi} \varrho_{0}, \varepsilon(x, y, \xi, \eta, t)\right. \\
& \left.+\partial_{\xi^{\prime}} \varrho_{t_{0}, \varepsilon}\left(x^{\prime}, y, \xi^{\prime}, \eta, t\right) \varrho_{t_{0}, \varepsilon}(x, y, \xi, \eta, t)\right] d x^{\prime} d \xi^{\prime} d x d \xi d y d \eta
\end{aligned}
$$

Now

$$
\begin{aligned}
& 2 \int f\left(x^{\prime}, \xi^{\prime}, t\right) \partial_{\xi^{\prime}} \varrho_{\varepsilon}\left(x^{\prime}, y, \xi^{\prime}, \eta, t\right) \varrho_{\varepsilon}(x, y, \xi, \eta, t) m(x, \xi, t) d x^{\prime} d \xi^{\prime} d x d \xi d y d \eta \\
& =-2 \int\left(\delta\left(\xi^{\prime}\right)-\nu\left(x^{\prime}, \xi^{\prime}, t\right)\right) \varrho_{\varepsilon}\left(x^{\prime}, y, \xi^{\prime}, \eta, t\right) \varrho_{\varepsilon}(x, y, \xi, \eta, t) m(x, \xi, t) d x^{\prime} d \xi^{\prime} d x d \xi d y d \eta \\
& =-2 \int \varrho_{\varepsilon}\left(x^{\prime}, y, 0, \eta, t\right) \varrho_{\varepsilon}(x, y, \xi, \eta, t) m(x, \xi, t) d x^{\prime} d x d \xi d y d \eta \\
& +2 \int \nu\left(x^{\prime}, \xi^{\prime}, t\right) \varrho_{\varepsilon}\left(x^{\prime}, y, \xi^{\prime}, \eta, t\right) \varrho_{\varepsilon}(x, y, \xi, \eta, t) m(x, \xi, t) d x^{\prime} d \xi^{\prime} d x d \xi d y d \eta \\
& \geq-2 \int \varrho_{\varepsilon}\left(x^{\prime}, y, 0, \eta, t\right) \varrho_{\varepsilon}(x, y, \xi, \eta, t) m(x, \xi, t) d x^{\prime} d x d \xi d y d \eta .
\end{aligned}
$$


In view of Lemma 4.4, there exists a null $\mathcal{N} \subseteq[0, T]$ with $0 \notin \mathcal{N}$ such that, for every $t_{0} \geq 0$ and all $(s, t) \in \Delta\left(\left[t_{0}, T\right] \backslash \mathcal{N}\right)$,

$$
\begin{aligned}
& 2 \int_{s}^{t} \int \varrho_{t_{0}, \varepsilon}\left(x^{\prime}, y, 0, \eta, r\right) \varrho_{t_{0}}(x, y, \xi, \eta, r) m(x, \xi, r) d x d \xi d x^{\prime} d y d \eta d r \\
& =-\int \operatorname{sgn}^{\varepsilon}(\eta)\left(\left(f * \varrho_{t_{0}, \varepsilon}\right)(y, \eta, t)-\left(f * \varrho_{t_{0}, \varepsilon}\right)(y, \eta, s)\right) d y d \eta+\int_{s}^{t} \operatorname{Err}^{(2)}\left(t_{0}, r, \varepsilon\right) d r
\end{aligned}
$$

with

$$
\begin{aligned}
\operatorname{Err}^{(2)}\left(t_{0}, t, \varepsilon\right)=\int m(x, \xi, t) \operatorname{sgn}\left(\xi^{\prime}\right) & \left(\partial_{\xi^{\prime}} \varrho_{t_{0}, \varepsilon}\left(x^{\prime}, y, \xi^{\prime}, \eta, t\right) \varrho_{t_{0}, \varepsilon}(x, y, \xi, \eta, t)\right. \\
& \left.+\varrho_{t_{0}, \varepsilon}\left(x^{\prime}, y, \xi^{\prime}, \eta, t\right) \partial_{\xi} \varrho_{t_{0}, \varepsilon}(x, y, \xi, \eta, t)\right) d y d \eta d x^{\prime} d \xi^{\prime} d x d \xi .
\end{aligned}
$$

Hence, for all $t_{0} \geq 0$ and all $s, t \in \Delta\left(\left[t_{0}, T\right] \backslash \mathcal{N}\right)$,

$$
\begin{aligned}
& \int\left(f * \varrho_{t_{0}, \varepsilon}\right)^{2}(y, \eta, t) d y d \eta-\int\left(f * \varrho_{t_{0}, \varepsilon}\right)^{2}(y, \eta, s) d y d \eta \\
\geq & \int \operatorname{sgn}^{\varepsilon}(\eta)\left[\left(f * \varrho_{t_{0}, \varepsilon}\right)(y, \eta, t)-\left(f * \varrho_{t_{0}, \varepsilon}\right)(y, \eta, s)\right] d y d \eta+\int_{s}^{t}\left[\operatorname{Err}^{(1)}\left(t_{0}, r, \varepsilon\right)-\operatorname{Err}^{(2)}\left(t_{0}, r, \varepsilon\right)\right] d r .
\end{aligned}
$$

It follows from Lemma B.1 that

$$
\begin{aligned}
& \int_{s}^{t}\left[\operatorname{Err}^{(1)}\left(t_{0}, r, \varepsilon\right)-\operatorname{Err}^{(2)}\left(t_{0}, r, \varepsilon\right)\right] d r \\
= & -2 \int\left(f\left(x^{\prime}, \xi^{\prime}, t\right)+\operatorname{sgn}\left(\xi^{\prime}\right)\right) m(x, \xi, t)\left[\varrho_{t_{0}, \varepsilon}\left(x^{\prime}, y, \xi^{\prime}, \eta, t\right) \partial_{\xi} \varrho_{t_{0}, \varepsilon}(x, y, \xi, \eta, t)\right. \\
& \left.+\partial_{\xi^{\prime}} \varrho_{t_{0}, \varepsilon}\left(x^{\prime}, y, \xi^{\prime}, \eta, t\right) \varrho_{t_{0}, \varepsilon}(x, y, \xi, \eta, t)\right] d x^{\prime} d \xi^{\prime} d x d \xi d y d \eta \\
\geq & -4 \int_{s}^{t} \int m(x, \xi, r)\left(\int \mid \int\left[\varrho_{t_{0}, \varepsilon}\left(x^{\prime}, y, \xi^{\prime}, \eta, r\right) \partial_{\xi} \varrho_{t_{0}, \varepsilon}(x, y, \xi, \eta, r)\right.\right. \\
& \left.\left.+\partial_{\xi^{\prime}} \varrho_{t_{0}, \varepsilon}\left(x^{\prime}, y, \xi^{\prime}, \eta, r\right) \varrho_{t_{0}, \varepsilon}(x, y, \xi, \eta, r)\right] d y d \eta \mid d x^{\prime} d \xi^{\prime}\right) d x d \xi d r \\
\geq & -C\left(t-t_{0}\right)^{\alpha} \int_{s}^{t} \int m(x, \xi, r) d x d \xi d r,
\end{aligned}
$$

and, thus,

$$
\begin{aligned}
& \int\left[\left(f * \varrho_{t_{0}, \varepsilon}\right)^{2}(y, \eta, t)-\left(f * \varrho_{t_{0}, \varepsilon}\right)^{2}(y, \eta, s)\right] d y d \eta \\
& \left.\geq \int \operatorname{sgn}^{\varepsilon}(\eta)\left[\left(f * \varrho_{t_{0}, \varepsilon}\right)(y, \eta, t)-f * \varrho_{t_{0}, \varepsilon}\right)(y, \eta, s)\right] d y d \eta-C\left(t-t_{0}\right)^{\alpha} \int_{s}^{t} \int m(x, \xi, r) d x d \xi d r .
\end{aligned}
$$

Letting $\varepsilon \rightarrow 0$ and using (4.3) yields, for all $t_{0} \geq 0$ and $(s, t) \in \Delta\left(\left[t_{0}, T\right] \backslash \mathcal{N}\right)$,

$$
\int\left[f^{2}(y, \eta, t)-|f|(y, \eta, t) \mid\right] d y d \eta \geq \int\left[f^{2}(y, \eta, s)-|f|(y, \eta, s) \mid\right] d y d \eta-C\left(t-t_{0}\right)^{\alpha} \int_{s}^{t} \int m(x, \xi, r) d x d \xi d r
$$


Fix now $t_{0} \geq 0$ and $(s, t) \in \Delta\left(\left[t_{0}, T\right] \backslash \mathcal{N}\right)$. For every partition $\Delta=\left\{s=t_{0} \leq \cdots \leq t_{m}=t\right\} \subseteq$ $\left[t_{0}, T\right] \backslash \mathcal{N}$ we have

$$
\begin{aligned}
& \int\left[f^{2}(x, \xi, t)-|f|(x, \xi, t)\right] d x d \xi-\int\left[f^{2}(x, \xi, s)-|f|(x, \xi, s)\right] d x d \xi \\
= & \sum_{i=1}^{N} \int\left[f^{2}\left(x, \xi, t_{i+1}\right)-|f|\left(x, \xi, t_{i+1}\right)\right] d x d \xi-\int\left[f^{2}\left(x, \xi, t_{i}\right)-|f|\left(x, \xi, t_{i}\right)\right] d x d \xi \\
\geq & -C \sum_{i=1}^{N}\left(t_{i+1}-t_{i}\right)^{\alpha} \int_{t_{i}}^{t_{i+1}} \int m(x, \xi, r) d x d \xi d r \\
\geq & -\|\Delta\|^{\alpha} C \int_{s}^{t} \int m(x, \xi, r) d x d \xi d r .
\end{aligned}
$$

Letting $\|\Delta\| \rightarrow 0$ yields, for all $t_{0} \geq 0$ and $(s, t) \in \Delta\left(\left[t_{0}, T\right] \backslash \mathcal{N}\right)$,

$$
\int\left[f^{2}(x, \xi, t)-|f|(x, \xi, t)\right] d x d \xi \geq \int\left[f^{2}(x, \xi, s)-|f|(x, \xi, s)\right] d x d \xi,
$$

and, since $0 \notin \mathcal{N}$ and $\int\left(f^{2}(x, \xi, 0)-|f|(x, \xi, 0)\right) d x d \xi=0$, for all $t \in[0, T] \backslash \mathcal{N}$ we conclude that (4.12) holds.

Proof of Theorem 4.1. The proof of Theorem 4.1 now becomes a simple consequence of all the previous analysis. Indeed Theorem 4.8 yields a generalized pathwise rough entropy/kinetic solution $f$ to (1.1) and Proposition 4.9 implies the existence of some $u \in L^{\infty}\left([0, T] ; L^{1}\left(\mathbb{R}^{N}\right)\right)$ such that $f(x, \xi, t)=$ $\chi(u(t, x), \xi)$. It then follows that $u \in L^{\infty}\left([0, T] ; L^{1}\left(\mathbb{R}^{N}\right)\right)$ is a pathwise rough entropy/kinetic solution to (1.1).

\section{Appendix A. Definitions And some estimates from Rough Paths theory}

We briefly recall the elements of rough paths theory used in this paper and for more details we refer to [FV10]. As already mentioned in the introduction, we use the theory of rough paths to define a topology which is stronger than the usual sup-norm on the space of driving signals. In the language of the rough path theory ordinary differential equations (ODE for short) are called controlled ODE and the signals are the controls. It is not difficult to construct examples showing that controlled ODE do not, in general, depend continuously on the path of the signal and that is necessary to use higher order iterated integrals of the controls.

This motivates the definition, for $x \in C^{1-\operatorname{var}}\left([0, T] ; \mathbb{R}^{N}\right)$, of the step $M$ signature

$$
S_{M}(x)_{0, T}=\left(1, \int_{0<u<T} d x_{u}, \ldots, \int_{0<u_{1}<\cdots<u_{M}<T} d x_{u_{1}} \otimes \cdots \otimes d x_{u_{M}}\right)
$$

We note that $S_{M}(x)$ takes values in the so-called truncated step- $M$ tensor algebra

$$
T^{M}\left(\mathbb{R}^{N}\right)=\mathbb{R} \oplus \mathbb{R}^{N} \oplus\left(\mathbb{R}^{N} \otimes \mathbb{R}^{N}\right) \oplus \ldots \oplus\left(\mathbb{R}^{N}\right)^{\otimes M} .
$$

In fact, $S_{M}(x)$ takes values in the smaller set $G^{M}\left(\mathbb{R}^{N}\right) \subset T^{M}\left(\mathbb{R}^{N}\right)$ given by

$$
G^{M}\left(\mathbb{R}^{N}\right):=\left\{S_{M}(x)_{0,1}: x \in C^{1-\operatorname{var}}\left([0,1] ; \mathbb{R}^{N}\right)\right\} .
$$

On $G^{M}\left(\mathbb{R}^{N}\right)$ we introduce the Carnot-Caratheodory norm

$$
\|g\|:=\inf \left\{\int_{0}^{1}|d \gamma|: \gamma \in C^{1-\operatorname{var}}\left([0,1] ; \mathbb{R}^{N}\right) \text { and } S_{M}(\gamma)_{0,1}=g\right\},
$$


which gives rise to a so-called homogeneous metric on $G^{M}\left(\mathbb{R}^{N}\right)$. Alternatively, for any $g \in T^{M}\left(\mathbb{R}^{N}\right)$, we may set

$$
|g|=|g|_{T^{M}\left(\mathbb{R}^{N}\right)}=\max _{k=1 \ldots M}\left|\pi_{k}(g)\right|,
$$

where $\pi_{k}$ is the projection of $g$ onto the $k$-th tensor level. This defines an inhomogeneous metric on $G^{M}\left(\mathbb{R}^{N}\right)$. It turns out that the topologies induced by $\|\cdot\|$ and $|\cdot|$ are actually equivalent.

For paths in $T^{M}\left(\mathbb{R}^{N}\right)$ starting at the fixed point $e:=1+0+\ldots+0$ and $\beta \in(0,1]$, one may then define $\beta$-Hölder metrics, extending the usual metrics for paths in $\mathbb{R}^{N}$ starting at zero. The homogeneous $\beta$-Hölder metric is denoted by $d_{\beta-\text { Höl }}$ and the inhomogeneous one by $\rho_{\beta-\mathrm{Höl}}$. A corresponding norm is defined by $\|\cdot\|_{\beta-\text { Höl }}=d_{\beta-\text { Höl }}(\cdot, 0)$ where 0 denotes the constant $e$-valued path.

A geometric $\beta$-Hölder rough path $x$ is a path in $T^{\lfloor 1 / \beta\rfloor}\left(\mathbb{R}^{N}\right)$ which can be approximated by lifts of smooth paths in the $d_{\beta-\text { Höl }}$ metric. It can be shown that rough paths actually take values in $G^{\lfloor 1 / \beta\rfloor}\left(\mathbb{R}^{N}\right)$. We denote by $C_{0}^{0, \beta-\text { Höl }}\left([0, T], G^{\lfloor 1 / \beta\rfloor}\left(\mathbb{R}^{N}\right)\right)$ the space of geometric $\beta$-Hölder rough paths.

We next recall some basic stability estimates for solutions to rough differential equations (RDE for short). For definiteness we consider RDE of the form

$$
d x=V(x) \circ d z,
$$

where $z$ is a geometric $\alpha$-Hölder rough path. It is well known (see, for example, [FV10]) that the RDE above has a flow $\psi^{z}$ of solutions. The following is taken from [CDFO13, Lemma 13].

Lemma A.1. Let $\alpha \in(0,1), \gamma>\frac{1}{\alpha} \geq 1, k \in \mathbb{N}$ and assume that $V \in \operatorname{Lip}^{\gamma+k}\left(\mathbb{R}^{N} ; \mathbb{R}^{N}\right)$. For all $R>0$ there exist $C=C\left(R,\|V\|_{\mathrm{Lip}^{\gamma+k}}\right)$ and $K=K\left(R,\|V\|_{\mathrm{Lip}^{\gamma+k}}\right)$, which are non-decreasing in all arguments, such that, for all geometric $\alpha$-Hölder rough paths $z^{1}, z^{2} \in C_{0}^{\alpha-\mathrm{Höl}}\left([0, T] ; G^{\left[\frac{1}{\alpha}\right]}\left(\mathbb{R}^{N}\right)\right)$ with $\left\|z^{1}\right\|_{\alpha-\mathrm{Höl} ;[0, T]},\left\|z^{2}\right\|_{\alpha-\mathrm{Höl} ;[0, T]} \leq R$ and all $n \in\{0, \ldots, k\}$,

$$
\begin{array}{r}
\sup _{x \in \mathbb{R}^{N}}\left\|D^{n}\left(\psi^{z^{1}}-\psi^{z^{2}}\right)(x)\right\|_{\alpha-\mathrm{Höl} ;[0, T]} \leq C \rho_{\alpha-\mathrm{Höl;} ;[0, T]}\left(z^{1}, z^{2}\right), \\
\sup _{x \in \mathbb{R}^{N}}\left\|D^{n}\left(\left(\psi^{z^{1}}\right)^{-1}-\left(\psi^{z^{2}}\right)^{-1}\right)(x)\right\|_{\alpha-\mathrm{Höl} ;[0, T]} \leq C \rho_{\alpha-\mathrm{Höl} ;[0, T]}\left(z^{1}, z^{2}\right)
\end{array}
$$

and, for all $n \in\{1, \ldots, k\}$,

$$
\sup _{x \in \mathbb{R}^{N}}\left\|D^{n} \psi^{z^{1}}(x)\right\|_{\alpha-\mathrm{Höl} ;[0, T]} \leq K \quad \text { and } \sup _{x \in \mathbb{R}^{N}}\left\|D^{n}\left(\psi^{z^{1}}\right)^{-1}(x)\right\|_{\alpha-\mathrm{Hö̈} ;[0, T]} \leq K .
$$

\section{Appendix B. Convolution error estimates}

Now we present the proof of the error estimate.

Lemma B.1. Let $\varrho_{t_{0}, \varepsilon}$ be as in (3.4). For each $R>0$ there exists $C=C(R)>0$ such that, for all $t \in[0, T]$ and all $\alpha$-Hölder rough paths $z$ with $\|z\|_{\alpha-\mathrm{Höl} ;[0, T]} \leq R$,

$$
\begin{aligned}
\sup _{(x, \xi) \in \mathbb{R}^{N+1}} \int & \mid \int \varrho_{t_{0}, \varepsilon}\left(x^{\prime}, y, \xi^{\prime}, \eta, r\right) \partial_{\xi} \varrho_{t_{0}, \varepsilon}(x, y, \xi, \eta, r) \\
& +\partial_{\xi^{\prime}} \varrho_{t_{0}, \varepsilon}\left(x^{\prime}, y, \xi^{\prime}, \eta, r\right) \varrho_{t_{0}, \varepsilon}(x, y, \xi, \eta, r) d y d \eta \mid d x^{\prime} d \xi^{\prime} \leq C\left(r-t_{0}\right)^{\alpha} .
\end{aligned}
$$


Proof. In what follows for simplicity we write $X_{\left(x^{\prime}, \xi^{\prime}\right)}$ for $X_{\left(r, x^{\prime}, \xi^{\prime}\right)}\left(r-t_{0}\right)$. We have:

$$
\begin{aligned}
& \varrho_{t_{0}, \varepsilon}\left(x^{\prime}, y, \xi^{\prime}, \eta, r\right) \partial_{\xi} \varrho_{t_{0}, \varepsilon}(x, y, \xi, \eta, r)+\partial_{\xi^{\prime}} \varrho_{t_{0}, \varepsilon}\left(x^{\prime}, y, \xi^{\prime}, \eta, r\right) \varrho_{t_{0}, \varepsilon}(x, y, \xi, \eta, r) \\
& =\varrho_{\varepsilon}^{s}\left(X_{\left(x^{\prime}, \xi^{\prime}\right)}-y\right) \varrho_{\varepsilon}^{v}\left(\Xi_{\left(x^{\prime}, \xi^{\prime}\right)}-\eta\right) \partial_{\xi}\left[\varrho_{\varepsilon}^{s}\left(X_{(x, \xi)}-y\right) \varrho_{\varepsilon}^{v}\left(\Xi_{(x, \xi)}-\eta\right)\right] \\
& +\partial_{\xi^{\prime}}\left[\varrho_{\varepsilon}^{s}\left(X_{\left(x^{\prime}, \xi^{\prime}\right)}-y\right) \varrho_{\varepsilon}^{v}\left(\Xi_{\left(x^{\prime}, \xi^{\prime}\right)}-\eta\right)\right] \varrho_{\varepsilon}^{s}\left(X_{(x, \xi)}-y\right) \varrho_{\varepsilon}^{v}\left(\Xi_{(x, \xi)}-\eta\right) \\
& =-\varrho_{\varepsilon}^{s}\left(X_{\left(x^{\prime}, \xi^{\prime}\right)}-y\right) \varrho_{\varepsilon}^{v}\left(\Xi_{\left(x^{\prime}, \xi^{\prime}\right)}-\eta\right) \partial_{\xi} X_{(x, \xi)} \partial_{y} \varrho_{\varepsilon}^{s}\left(X_{(x, \xi)}-y\right) \varrho_{\varepsilon}^{v}\left(\Xi_{(x, \xi)}-\eta\right) \\
& -\partial_{\xi^{\prime}} X_{\left(x^{\prime}, \xi^{\prime}\right)} \partial_{y} \varrho_{\varepsilon}^{s}\left(X_{\left(x^{\prime}, \xi^{\prime}\right)}-y\right) \varrho_{\varepsilon}^{v}\left(\Xi_{\left(x^{\prime}, \xi^{\prime}\right)}-\eta\right) \varrho_{\varepsilon}^{s}\left(X_{(x, \xi)}-y\right) \varrho_{\varepsilon}^{v}\left(\Xi_{(x, \xi)}-\eta\right) \\
& -\varrho_{\varepsilon}^{s}\left(X_{\left(x^{\prime}, \xi^{\prime}\right)}-y\right) \varrho_{\varepsilon}^{v}\left(\Xi_{\left(x^{\prime}, \xi^{\prime}\right)}-\eta\right) \varrho_{\varepsilon}^{s}\left(X_{(x, \xi)}-y\right) \partial_{\xi} \Xi_{(x, \xi)} \partial_{\eta} \varrho_{\varepsilon}^{v}\left(\Xi_{(x, \xi)}-\eta\right) \\
& -\varrho_{\varepsilon}^{s}\left(X_{\left(x^{\prime}, \xi^{\prime}\right)}-y\right) \partial_{\xi^{\prime}} \Xi_{\left(x^{\prime}, \xi^{\prime}\right)} \partial_{\eta} \varrho_{\varepsilon}^{v}\left(\Xi_{\left(x^{\prime}, \xi^{\prime}\right)}-\eta\right) \varrho_{\varepsilon}^{s}\left(X_{(x, \xi)}-y\right) \varrho_{\varepsilon}^{v}\left(\Xi_{(x, \xi)}-\eta\right) .
\end{aligned}
$$

We next show the details for the first two terms, since the second two terms are treated similarly. An integration by parts yields

$$
\begin{aligned}
& -\int \varrho_{\varepsilon}^{s}\left(X_{\left(x^{\prime}, \xi^{\prime}\right)}-y\right) \varrho_{\varepsilon}^{v}\left(\Xi_{\left(x^{\prime}, \xi^{\prime}\right)}-\eta\right) \partial_{\xi} X_{(x, \xi)} \partial_{y} \varrho_{\varepsilon}^{s}\left(X_{(x, \xi)}-y\right) \varrho_{\varepsilon}^{v}\left(\Xi_{(x, \xi)}-\eta\right) d y \\
& =\int \partial_{y} \varrho_{\varepsilon}^{s}\left(X_{\left(x^{\prime}, \xi^{\prime}\right)}-y\right) \varrho_{\varepsilon}^{v}\left(\Xi_{\left(x^{\prime}, \xi^{\prime}\right)}-\eta\right) \partial_{\xi} X_{(x, \xi)} \varrho_{\varepsilon}^{s}\left(X_{(x, \xi)}-y\right) \varrho_{\varepsilon}^{v}\left(\Xi_{(x, \xi)}-\eta\right) d y
\end{aligned}
$$

and thus

$$
\begin{aligned}
& \int\left[-\varrho_{\varepsilon}^{s}\left(X_{\left(x^{\prime}, \xi^{\prime}\right)}-y\right) \varrho_{\varepsilon}^{v}\left(\Xi_{\left(x^{\prime}, \xi^{\prime}\right)}-\eta\right) \partial_{\xi} X_{(x, \xi)} \partial_{y} \varrho_{\varepsilon}^{s}\left(X_{(x, \xi)}-y\right) \varrho_{\varepsilon}^{v}\left(\Xi_{(x, \xi)}-\eta\right)\right. \\
& \left.-\partial_{\xi^{\prime}} X_{\left(x^{\prime}, \xi^{\prime}\right)} \partial_{y} \varrho_{\varepsilon}^{s}\left(X_{\left(x^{\prime}, \xi^{\prime}\right)}-y\right) \varrho_{\varepsilon}^{v}\left(\Xi_{\left(x^{\prime}, \xi^{\prime}\right)}-\eta\right) \varrho_{\varepsilon}^{s}\left(X_{(x, \xi)}-y\right) \varrho_{\varepsilon}^{v}\left(\Xi_{(x, \xi)}-\eta\right)\right] d y d \eta \\
= & \int \partial_{y} \varrho_{\varepsilon}^{s}\left(X_{\left(x^{\prime}, \xi^{\prime}\right)}-y\right) \varrho_{\varepsilon}^{s}\left(X_{(x, \xi)}-y\right) \varrho_{\varepsilon}^{v}\left(\Xi_{\left(x^{\prime}, \xi^{\prime}\right)}-\eta\right) \varrho_{\varepsilon}^{v}\left(\Xi_{(x, \xi)}-\eta\right) d y d \eta\left[\partial_{\xi} X_{(x, \xi)}-\partial_{\xi^{\prime}} X_{\left(x^{\prime}, \xi^{\prime}\right)}\right] \\
= & -\int\left[\varrho_{\varepsilon}^{s}\left(X_{\left(x^{\prime}, \xi^{\prime}\right)}-y\right) \partial_{y} \varrho_{\varepsilon}^{s}\left(X_{(x, \xi)}-y\right) \varrho_{\varepsilon}^{v}\left(\Xi_{\left(x^{\prime}, \xi^{\prime}\right)}-\eta\right) \varrho_{\varepsilon}^{v}\left(\Xi_{(x, \xi)}-\eta\right)\right] d y d \eta\left[\partial_{\xi} X_{(x, \xi)}-\partial_{\xi^{\prime}} X_{\left(x^{\prime}, \xi^{\prime}\right)}\right] .
\end{aligned}
$$

Fix some $K>0$ and set

$$
\mathcal{A}:=\left\{x, x^{\prime} \in \mathbb{R}^{N}, \xi, \xi^{\prime} \in \mathbb{R}:\left|X_{\left(x^{\prime}, \xi^{\prime}\right)}-X_{(x, \xi)}\right| \leq K \varepsilon \text { and }\left|\Xi_{\left(x^{\prime}, \xi^{\prime}\right)}-\Xi_{(x, \xi)}\right| \leq K \varepsilon\right\} .
$$

Then

$$
\begin{aligned}
& \mid \int\left[-\varrho_{\varepsilon}^{s}\left(X_{\left(x^{\prime}, \xi^{\prime}\right)}-y\right) \varrho_{\varepsilon}^{v}\left(\Xi_{\left(x^{\prime}, \xi^{\prime}\right)}-\eta\right) \partial_{\xi} X_{(x, \xi)} \partial_{y} \varrho_{\varepsilon}^{s}\left(X_{(x, \xi)}-y\right) \varrho_{\varepsilon}^{v}\left(\Xi_{(x, \xi)}-\eta\right)\right. \\
& \left.-\partial_{\xi^{\prime}} X_{\left(x^{\prime}, \xi^{\prime}\right)} \partial_{y} \varrho_{\varepsilon}^{s}\left(X_{\left(x^{\prime}, \xi^{\prime}\right)}-y\right) \varrho_{\varepsilon}^{v}\left(\Xi_{\left(x^{\prime}, \xi^{\prime}\right)}-\eta\right) \varrho_{\varepsilon}^{s}\left(X_{(x, \xi)}-y\right) \varrho_{\varepsilon}^{v}\left(\Xi_{(x, \xi)}-\eta\right)\right] d y d \eta \mid \\
& \leq \sup _{\mathcal{A}}\left|\partial_{\xi} X_{(x, \xi)}-\partial_{\xi^{\prime}} X_{\left(x^{\prime}, \xi^{\prime}\right)}\right| \int \varrho_{\varepsilon}^{s}\left(X_{\left(x^{\prime}, \xi^{\prime}\right)}-y\right)\left|\partial_{y} \varrho_{\varepsilon}^{s}\left(X_{(x, \xi)}-y\right)\right| \varrho_{\varepsilon}^{s}\left(\Xi_{\left(x^{\prime}, \xi^{\prime}\right)}-\eta\right) \varrho_{\varepsilon}^{s}\left(\Xi_{(x, \xi)}-\eta\right) d y d \eta .
\end{aligned}
$$

Next we note that

$$
\begin{aligned}
& \int \varrho_{\varepsilon}^{s}\left(X_{\left(x^{\prime}, \xi^{\prime}\right)}-y\right)\left|\partial_{y} \varrho_{\varepsilon}^{s}\left(X_{(x, \xi)}-y\right)\right| \varrho_{\varepsilon}^{s}\left(\Xi_{\left(x^{\prime}, \xi^{\prime}\right)}-\eta\right) \varrho_{\varepsilon}^{s}\left(\Xi_{(x, \xi)}-\eta\right) d y d \eta d x^{\prime} d \xi^{\prime} \\
& =\int \varrho_{\varepsilon}^{s}\left(x^{\prime}-y\right)\left|\partial_{y} \varrho_{\varepsilon}^{s}\left(X_{(x, \xi)}-y\right)\right| \varrho_{\varepsilon}^{s}\left(\xi^{\prime}-\eta\right) \varrho_{\varepsilon}^{s}\left(\Xi_{(x, \xi)}-\eta\right) d y d \eta d x^{\prime} d \xi^{\prime} \\
& =\int\left|\partial_{y} \varrho_{\varepsilon}^{s}\left(X_{(x, \xi)}-y\right)\right| \varrho_{\varepsilon}^{s}\left(\Xi_{(x, \xi)}-\eta\right) d y d \eta \\
& =\int\left|\partial_{y} \varrho_{\varepsilon}^{s}\left(X_{\left(x^{\prime}, \xi^{\prime}\right)}-y\right)\right| d y \leq \frac{K}{\varepsilon}
\end{aligned}
$$


Thus

$$
\begin{aligned}
& \int \mid \int-\varrho_{\varepsilon}^{s}\left(X_{\left(x^{\prime}, \xi^{\prime}\right)}-y\right) \varrho_{\varepsilon}^{v}\left(\Xi_{\left(x^{\prime}, \xi^{\prime}\right)}-\eta\right) \partial_{\xi} X_{(x, \xi)} \partial_{y} \varrho_{\varepsilon}^{s}\left(X_{(x, \xi)}-y\right) \varrho_{\varepsilon}^{v}\left(\Xi_{(x, \xi)}-\eta\right) \\
& -\partial_{\xi^{\prime}} X_{\left(x^{\prime}, \xi^{\prime}\right)} \partial_{y} \varrho_{\varepsilon}^{s}\left(X_{\left(x^{\prime}, \xi^{\prime}\right)}-y\right) \varrho_{\varepsilon}^{v}\left(\Xi_{\left(x^{\prime}, \xi^{\prime}\right)}-\eta\right) \varrho_{\varepsilon}^{s}\left(X_{(x, \xi)}-y\right) \varrho_{\varepsilon}^{v}\left(\Xi_{(x, \xi)}-\eta\right) d y d \eta \mid d x^{\prime} d \xi^{\prime} \\
\leq & \frac{K}{\varepsilon} \sup _{\mathcal{A}}\left|\partial_{\xi} X_{(x, \xi)}-\partial_{\xi^{\prime}} X_{\left(x^{\prime}, \xi^{\prime}\right)}\right| .
\end{aligned}
$$

From Lemma A.1 we know that

$$
C:=\sup _{x, \xi}\left\|D^{2}\left(\begin{array}{c}
X_{(r, x, \xi)}(\cdot) \\
\Xi_{(r, x, \xi)}(\cdot)
\end{array}\right)\right\|_{\alpha-\mathrm{Höl} ;[0, r]}<\infty .
$$

Moreover, $D^{2}\left(\begin{array}{c}X_{(r, x, \xi)}(0) \\ \Xi_{(r, x, \xi)}(0)\end{array}\right)=0$ for all $(r, x, \xi)$. Thus,

$$
\begin{aligned}
\sup _{x, \xi}\left\|D^{2}\left(\begin{array}{c}
X_{(r, x, \xi)}(t) \\
\Xi_{(r, x, \xi)}(t)
\end{array}\right)\right\| & =\sup _{x, \xi}\left\|D^{2}\left(\begin{array}{c}
X_{(r, x, \xi)}(t) \\
\Xi_{(r, x, \xi)}(t)
\end{array}\right)-D^{2}\left(\begin{array}{c}
X_{(r, x, \xi)}(0) \\
\Xi_{(r, x, \xi)}(0)
\end{array}\right)\right\| \\
& \leq t^{\alpha} \sup _{x, \xi}\left\|D^{2}\left(\begin{array}{c}
X_{(r, x, \xi)}(\cdot) \\
\Xi_{(r, x, \xi)}(\cdot)
\end{array}\right)\right\|_{\alpha-\text { Höl; } ; 0, r]} \\
& =C t^{\alpha} .
\end{aligned}
$$

We conclude that there exists a uniform constant $C>0$ such that, for all $x, \xi, x^{\prime}, \xi^{\prime}$,

$$
\begin{aligned}
& \left|\partial_{\xi} X_{(r, x, \xi)}\left(r-t_{0}\right)-\partial_{\xi^{\prime}} X_{\left(r, x^{\prime}, \xi^{\prime}\right)}\left(r-t_{0}\right)\right| \\
& +\left|\partial_{\xi} \Xi_{(r, x, \xi)}\left(r-t_{0}\right)-\partial_{\xi^{\prime}} \Xi_{\left(r, x^{\prime}, \xi^{\prime}\right)}\left(r-t_{0}\right)\right| \leq C\left(r-t_{0}\right)^{\alpha}\left\|\begin{array}{c}
x-x^{\prime} \\
\xi-\xi^{\prime}
\end{array}\right\| .
\end{aligned}
$$

Again by Lemma A.1 we have that

$$
C:=\sup _{y, \eta}\left\|D\left(\begin{array}{c}
Y_{(s, y, \eta)}(\cdot) \\
\zeta_{(s, y, \eta)}(\cdot)
\end{array}\right)\right\|_{\alpha-\mathrm{Höl} ;[s, t]}<\infty,
$$

and, since $\left(\begin{array}{c}Y_{\left(t_{0}, y, \eta\right)}(r) \\ \zeta_{\left(t_{0}, y, \eta\right)}(r)\end{array}\right)$ is the inverse of $\left(\begin{array}{c}X_{(r, x, \xi)}\left(r-t_{0}\right) \\ \Xi_{(r, x, \xi)}\left(r-t_{0}\right)\end{array}\right)$, it follows that,

$$
\text { if }\left\|\begin{array}{c}
X_{\left(x^{\prime}, \xi^{\prime}\right)}-X_{(x, \xi)} \\
\Xi_{\left(x^{\prime}, \xi^{\prime}\right)}-\Xi_{(x, \xi)}
\end{array}\right\| \leq K \varepsilon \text {, then }\left\|\begin{array}{c}
x-x^{\prime} \\
\xi-\xi^{\prime}
\end{array}\right\| \leq C \varepsilon .
$$

Hence,

$\sup _{\mathcal{A}}\left[\left|\partial_{\xi} X_{(r, x, \xi)}\left(r-t_{0}\right)-\partial_{\xi^{\prime}} X_{\left(r, x^{\prime}, \xi^{\prime}\right)}\left(r-t_{0}\right)\right|+\left|\partial_{\xi} \Xi_{(r, x, \xi)}\left(r-t_{0}\right)-\partial_{\xi^{\prime}} \Xi_{\left(r, x^{\prime}, \xi^{\prime}\right)}\left(r-t_{0}\right)\right|\right] \leq C\left(r-t_{0}\right)^{\alpha} \varepsilon$, which, in light of $(\overline{\mathrm{B} .2})$, finishes the proof. Note that the constant $C=C(R)$ may be chosen uniformly for all $\alpha$-Hölder rough paths $z$ with $\|z\|_{\alpha-\mathrm{Höl} ;[0, T]} \leq R$.

\section{REFERENCES}

[CDFO13] D. Crisan, J. Diehl, P. K. Friz, and H. Oberhauser, Robust filtering: correlated noise and multidimensional observation, Ann. Appl. Probab. 23 (2013), no. 5, 2139-2160. A

[CDK12] Gui-Qiang Chen, Qian Ding, and Kenneth H. Karlsen, On nonlinear stochastic balance laws, Arch. Ration. Mech. Anal. 204 (2012), no. 3, 707-743. 1]

[Dal06] Anne-Laure Dalibard, Kinetic formulation for heterogeneous scalar conservation laws, Ann. Inst. H. Poincaré Anal. Non Linéaire 23 (2006), 475-498. 2] 20

[DHV13] Arnaud Debussche, Martina Hofmanová, and Julien Vovelle, Degenerate parabolic stochastic partial differential equations: Quasilinear case, preprint (2013), 1-34. 1] 
[DV10] A. Debussche and J. Vovelle, Scalar conservation laws with stochastic forcing, J. Funct. Anal. 259 (2010), no. 4, 1014-1042. 1

[FG14] Peter Friz and Benjamin Gess, Stochastic scalar conservation laws driven by rough paths, arXiv:1403.6785 (2014), 1-29. 1

[FN08] Jin Feng and David Nualart, Stochastic scalar conservation laws, J. Funct. Anal. 255 (2008), no. 2, $313-373$. 1

[FV10] Peter K. Friz and Nicolas B. Victoir, Multidimensional stochastic processes as rough paths, Cambridge Studies in Advanced Mathematics, vol. 120, Cambridge University Press, Cambridge, 2010, Theory and applications. 2. A

[Hof13] Martina Hofmanová, Degenerate parabolic stochastic partial differential equations, Stochastic Process. Appl. 123 (2013), no. 12, 4294-4336. 1]

[LL06a] Jean-Michel Lasry and Pierre-Louis Lions, Jeux à champ moyen. I. Le cas stationnaire, C. R. Math. Acad. Sci. Paris 343 (2006), no. 9, 619-625. 1

[LL06b] _ Jeux à champ moyen. II. Horizon fini et contrôle optimal, C. R. Math. Acad. Sci. Paris 343 (2006), no. 10, 679-684. [1]

[LL07] _ ㄴ. Mean field games, Jpn. J. Math. 2 (2007), no. 1, 229-260. 1

[LPS13] Pierre-Louis Lions, Benoît Perthame, and Panagiotis E. Souganidis, Scalar conservation laws with rough (stochastic) fluxes, Stochastic Partial Differential Equations: Analysis and Computations 1 (2013), no. 4, 664-686. 1] 1, 2] [3]

[LPS14] Scalar conservation laws with rough (stochastic) fluxes; the spatially dependent case, arXiv:1403.4424 (2014), 1-17. 1, 1 2, 3

[LS98a] Pierre-Louis Lions and Panagiotis E. Souganidis, Fully nonlinear stochastic partial differential equations, C. R. Acad. Sci. Paris Sér. I Math. 326 (1998), no. 9, 1085-1092. 1

[LS98b] _ Fully nonlinear stochastic partial differential equations: non-smooth equations and applications, C. R. Acad. Sci. Paris Sér. I Math. 327 (1998), no. 8, 735-741. 1

[LS00a] Fully nonlinear stochastic PDE with semilinear stochastic dependence, C. R. Acad. Sci. Paris Sér. I Math. 331 (2000), no. 8, 617-624. 1

[LS00b] U Uniqueness of weak solutions of fully nonlinear stochastic partial differential equations, C. R. Acad. Sci. Paris Sér. I Math. 331 (2000), no. 10, 783-790. 1

[LS02] ㄴ. Viscosity solutions of fully nonlinear stochastic partial differential equations, Sūrikaisekikenkyūsho Kōkyūroku (2002), no. 1287, 58-65, Viscosity solutions of differential equations and related topics (Japanese) (Kyoto, 2001). 1

[Per02] Benoît Perthame, Kinetic formulation of conservation laws, Oxford Lecture Series in Mathematics and its Applications, vol. 21, Oxford University Press, Oxford, 2002. 1, 2, 2, 4, 4, 4,

Department of Mathematics, University of Chicago, Chicago, IL 60637, USA

E-mail address: gess@uchicago.edu

Department of Mathematics, University of Chicago, Chicago, IL 60637, USA

E-mail address: souganidis@math.uchicago.edu 